Article

\title{
Tumor Extracellular Vesicles Regulate Macrophage-Driven Metastasis through CCL5
}

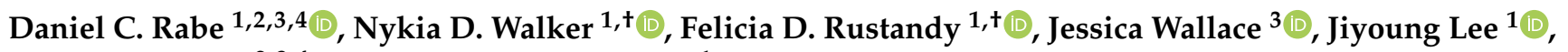 \\ Shannon L. Stott ${ }^{2,3,4, *}$ and Marsha Rich Rosner ${ }^{1, *}$ \\ 1 Ben May Department for Cancer Research, University of Chicago, Chicago, IL 60615, USA; \\ drabe@mgh.harvard.edu (D.C.R.); nykia.d.walker@gmail.com (N.D.W.); feliciarustandy@gmail.com (F.D.R.); \\ jiyounglee@gwu.edu (J.L.) \\ 2 Center for Cancer Research, Massachusetts General Hospital, Boston, MA 02129, USA \\ 3 BioMEMS Resource Center, Center for Engineering in Medicine and Surgical Services, Massachusetts General \\ Hospital and Harvard Medical School, Boston, MA 02129, USA; jessica.wallace.100@comcast.net \\ 4 Broad Institute of MIT and Harvard, Cambridge, MA 02142, USA \\ * Correspondence: sstott@mgh.harvard.edu (S.L.S.); mrosner@uchicago.edu (M.R.R.); \\ Tel.: +1-(617)-643-2658 (S.L.S.); +1-(773)-702-0380 (M.R.R.) \\ + Authors contributed equally to this work.
}

check for updates

Citation: Rabe, D.C.; Walker, N.D.; Rustandy, F.D.; Wallace, J.; Lee, J.; Stott, S.L.; Rosner, M.R. Tumor Extracellular Vesicles Regulate Macrophage-Driven Metastasis through CCL5. Cancers 2021, 13, 3459. https://doi.org/10.3390/cancers 13143459

Academic Editors: Eishi Ashihara and Asako Yamayoshi

Received: 2 June 2021

Accepted: 4 July 2021

Published: 10 July 2021

Publisher's Note: MDPI stays neutral with regard to jurisdictional claims in published maps and institutional affiliations.

Copyright: (c) 2021 by the authors. Licensee MDPI, Basel, Switzerland. This article is an open access article distributed under the terms and conditions of the Creative Commons Attribution (CC BY) license (https:// creativecommons.org/licenses/by/ $4.0 /)$.
Simple Summary: About 10-20 percent of patients with breast cancer are diagnosed with triplenegative breast cancer (TNBC). These tumors are named for their lack of expression of estrogen receptor (ER), progesterone receptor (PR), and amplification of human epidermal growth factor receptor 2 (HER2). These genes are targeted by therapies in other breast cancer patients. However, most TNBC patients recur within 5 years. Understanding how and why these tumors metastasize will help clinicians better treat these underserved cancer patients. TNBC tumors are highly infiltrated by tumor-associated macrophages (TAMs) that promote tumorigenesis and metastasis. Our study elucidates how the tumor co-opts macrophages recruited to the tumor through extracellular vesicles (EVs), further increasing tumor metastasis. Expression of tumor CCL5 regulates EV secretion and cargo that further alters macrophage phenotype to drive tumor metastasis. Together, our data suggest a more extensive role of EVs in the biology of tumor metastasis as well as their potential use as biomarkers.

Abstract: Purpose: To understand how tumor cells alter macrophage biology once they are recruited to triple-negative breast cancer (TNBC) tumors by CCL5. Method: Mouse bone marrow derived macrophage (BMDMs) were isolated and treated with recombinant CCL5 protein alone, with tumor cell conditioned media, or with tumor extracellular vesicles (EVs). Media from these tumor EVeducated macrophages (TEMs) was then used to determine how these macrophages affect TNBC invasion. To understand the mechanism, we assayed the cytokine secretion from these macrophages to determine how they impact tumor cell invasion. Tumor CCL5 expression was varied in tumors to determine its role in regulating macrophage biology through EVs. Results: Tumor EVs are a necessary component for programming naïve macrophages toward a pro-metastatic phenotype. CCL5 expression in the tumor cells regulates both EV biogenesis/secretion/cargo and macrophage EV-education toward a pro-metastatic phenotype. Analysis of the tumor EV-educated macrophages (TEMs) showed secretion of a variety of factors including CXCL1, CTLA-4, IFNG, OPN, HGF, TGFB, and CCL19 capable of remodeling the surrounding tumor stroma and immune infiltrate. Injection of tumor cells with macrophages educated by metastatic tumor cell EVs into mice increased tumor metastasis to the lung. Conclusion: These results demonstrate that tumor-derived EVs are key mediators of macrophage education and likely play a more complex role in modulating tumor therapeutic response by regulating the tumor immune infiltrate.

Keywords: metastasis; tumor-associated macrophages (TAMs); extracellular vesicles (EVs); exosomes; CCL5; tumor microenvironment (TME); triple-negative breast cancer (TNBC) 


\section{Introduction}

Of the women in the United States that develop breast cancer, 15-20\% will have basallike or triple-negative breast cancer (TNBC) [1]. Triple-negative tumors lack expression of estrogen receptor (ER), progesterone receptor (PR), and amplification of human epidermal receptor 2 (HER2) [1]. Clinical outcomes have improved for many patients with breast cancer, as high as $98 \%$ five-year survival rates in patients treated with targeted anti-ER or anti-HER2 therapies. However, patients with TNBC have five-year survival rates close to $24 \%$ [2], and this disproportionately affects African-American women and lower income women $[1,3]$. Thus, it is important to identify mechanisms that drive metastatic progression of TNBCs as well as potential targets for therapeutic treatment and biomarkers for response to therapy or progression.

Tumor cells are driven to metastasize in part through interaction with cells in the microenvironment. Tumor-associated macrophages (TAMs), generally characterized as alternatively activated macrophages, play an essential role in driving metastasis $[4,5]$. Previous studies showed that deleting Colony Stimulating Factor-1 (CSF-1/M-CSF) in the mouse mammary tumor virus-polyoma virus middle $\mathrm{T}$ antigen breast cancer mouse model (MMTV-PyMT) delayed metastatic tumor progression without altering mammary tumor growth [6]. Furthermore, liver cancer studies demonstrated that macrophage ablation enhanced the anti-tumor effects of targeted therapies [7]. Finally, the extent of TAM infiltration, determined by Cluster of Differentiation 163 (CD163) staining, correlates positively with TNBC tumors, negatively with less aggressive ER+ tumors, and positively with poor outcome within TNBC tumors [8]. Thus, pro-metastatic TAMs play a significant role in the progression of TNBC tumors and contribute to poor prognosis of patients.

TAMs are recruited to mammary tumors through induction of a variety of cytokines and chemokines including C-C Motif Chemokine Ligand 2 (CCL2), CCL3, CCL5, Colony Stimulating Facotor-1 (CSF-1/M-CSF), and Granulocyte-Macrophage Colony-Stimulating Factor (GM-CSF or CSF2) [9-13]. This interaction is further complicated by proteomic studies that identified multiple subsets of cytokine-secreting TAMs in vivo [14]. A number of studies have implicated CCL5 $[10,11]$ in the recruitment of macrophages to mammary tumors. Our previous study showed that CCL5 mediates macrophage recruitment to TNBCs, and the metastasis suppressor Raf Kinase Inhibitory Protein (RKIP) blocks this recruitment by inhibiting CCL5 expression in tumor cells and CCR5 expression in macrophages [11]. CCL5 has also been shown to play a role in the recruitment of T cells. Specifically, studies have shown that tumor-derived CCL5 can recruit T-regulatory cells (T-regs) into the tumor, leading to CD8+ T-cell apoptosis. Blockade of CCL5 leads to decreased tumor growth in immune competent models, suggesting that T-cells are required to reduce tumor bulk [15]. Additionally, stromal CCL5 and tumor CCR3 (but not CCR1 or CCR5) expression together are associated with poor outcome in TNBC patients [16]. Although factors such as CCL5 that enable recruitment of TAMs to mammary tumors have been identified, the process by which recruited macrophages are educated or polarized by tumor cells is not well-understood.

One possible mechanism connecting tumor and macrophage education is through EVs secreted from the tumor cells and taken up by the immune system. EVs are small micro-vesicles with a double lipid bilayer, secreted by most cell types, and of endocytic or membrane origin [17]. Small EVs (which include exosomes) are often between 40 to $150 \mathrm{~nm}$ and thought to be formed through invagination of the endosome membrane to form multi-vesicular bodies (MVBs) [18-20]. Alternative pathways of secretion for large EVs are thought to occur through exocytosis at the plasma membrane. EV markers include Alix1, CD9, CD63, CD81, and TSG-101 [17,21]. Knock-down screens of genes involved in EV secretion identified Rab27a as necessary for full secretion of EVs from cells. Rab27a is required for docking of MVBs at the plasma membrane [22]. The ESCRT complex additionally assists in MVB sorting to the membrane before being assisted by Rab proteins to dock and fuse with the plasma membrane via SNARE-mediated membrane fusion to 
release exosomes into the extra-cellular space [23]. The most widely used protein to block EV secretion in cells for in vivo studies is Rab27a $[17,22]$.

A number of studies suggest that small EVs, including exosomes, modulate stromal and immune response within the tumor microenvironment [24,25]. Tumor-derived EVs promote formation of pre-metastatic niches $[20,25]$ and drive progenitor cells within the bone marrow toward a pro-metastatic state [26]. Tumor-derived EVs stimulate myofibroblast differentiation [27], suppress immune response by impeding T cell activation [28], inhibit NK function [29], or potentiate regulatory T cell (T-reg) activity [30]. Stromal EVs induce tumor cell migration [31] and can mediate chemotherapy resistance in breast cancer cells [32]. EVs can also be taken up by macrophages, likely through clathrin-dependent phagocytosis [33-35]. However, direct evidence that EVs regulate naïve macrophage polarization, particularly in primary tumors, or their functional effect on macrophage-driven metastasis in vivo is lacking.

Our results indicate that tumor EVs alone can educate macrophages to a TAM phenotype that drives invasion and metastasis of TNBC. The absence of EVs, either through removal by ultracentrifugation or reduced secretion through Rab27a knockdown reduce the ability of tumors to program macrophages. Furthermore, we show that macrophage education occurs through an indirect mechanism involving stimulation of tumor cells by CCL5. CCL5 expression within the tumor increases the secretion of tumor EVs and alters the ability of EVs to educate macrophages. This results in TAMs that reflect the metastatic state of the tumor cells. Clinically, the genes we identified in this study are highly correlated to CCL5, and cluster in a subset of TNBC patient tumors within The Cancer Genome Atlas (TCGA) cohort. Taken together, these results indicate that tumor EVs play an integral role in education of non-resident macrophages to a pro-metastatic TAM phenotype in TNBCs, driving tumor metastasis.

\section{Materials and Methods}

\subsection{Cell Culture}

MDA-MB-231, 293T, and L929 were obtained from ATCC. MDA-MB-231 1833 [36] (referred to as BM1) cells were obtained from Andy Minn and E0771-LMB cells were obtained from Robin Anderson [37]. Numerous vials were frozen upon original receipt of the cells, and all work was done within 15 passages of the initially received lines. Late passage cells were sent to Idexx for cell line authentication using STR analysis and cells were routinely checked for mycoplasma contamination (Lonza, Basel, Switzerland). BM1, 293T, and LMB cell lines were cultured in high-glucose DMEM media with L-glutamine (Corning, Corning, NY, USA) supplemented with 10\% fetal bovine serum (Corning), and $50 \mathrm{U} / \mathrm{mL}$ penicillin and $50 \mathrm{\mu g} / \mathrm{mL}$ streptomycin (Gibco, Dublin, Ireland) and L929 grown in RPMI 1640 (Corning) with the same supplementation as above.

\subsection{Lentiviral Transductions}

All lentiviral work was done according to institutional biosafety rules, utilizing BSL3 practices and performed in a BSL2 hood. One million 293T cells were plated in a T-25 flask the evening prior. The following day, lentiviral vectors were incubated with 4th generation viral packaging vectors and Transit LT- 1 for 30 min as described by the manufacturer's protocol. DNA/LT-1 mixtures were then used to transfect 293T cells for viral production. Transfected cells were grown for $24-48 \mathrm{~h}$ prior to viral harvesting. After incubation, viral containing media was removed, centrifuged at $2000 \times g$ to removed dead cells and debris, then filtered through a $0.45 \mu \mathrm{m}$ PES syringe filter. Polybrene was added to media for a final concentration of $8 \mathrm{ng} / \mathrm{mL}$. Media was then added to target cells. Following a 24-h transduction period, cells were washed, trypsinized, and plated.

\section{3. shRNA Work}

Lentiviral shRNA plasmids targeting Rab27a from The RNAi consortium (TRC) were purchased from Open Biosystems. Lentiviruses were produced as described above. BM1 
cells were transduced and selected in $3 \mu \mathrm{g} / \mathrm{mL}$ of puromycin and LMB cells with $10 \mu \mathrm{g} / \mathrm{mL}$ of puromycin for 14 days. Five shRNA targets against Rab27a were used for both human and mouse Rab27a, with a non-targeting shRNA used as control.

\subsection{CRISPR-Cas9 Work}

Lentiviral all-in-one plasmids containing Cas9 as well as guide RNAs were purchased from Applied Biological Materials. Lentivirus was produced as described above. BM1 cells were then infected, then selected for 14 days using $3 \mu \mathrm{g} / \mathrm{mL}$ of puromycin. Transduced cells were assayed for CCL5 expression using a CCL5 ELISA from Ray Biotech as well as by qPCR. Media samples were concentrated $10 \times$ to ensure even low levels in KO cells could be measured.

For dCas9-KRAB knock-down and dCas9-VPR over-expression of Ccl5 in mouse LMB cells, sequences were determined using Broad's online sgRNA tool, and sgRNA sequences were ordered from IDT and inserted into sgRNA vectors obtained from Broad. Cells were first transduced with dCas9-KRAB or dCas9-VPR and selected with $10 \mu \mathrm{g} / \mathrm{mL}$ blasticidin followed by sgRNA transduction and selection with $10 \mu \mathrm{g} / \mathrm{mL}$ puromycin for 14 days each. RNA levels of Ccl5 were measured in cells and EVs using ddPCR with Gapdh as a loading control.

\section{5. qRT-PCR Primers}

Hs GAPDH-F: TGCACCACCACCTGCTTAGC

Hs GAPDH-R: GGCATGGACTGTGGTCATGAG

Hs CCL5-F: CCAGCAGTCGTCTTTGTCAC

Hs CCL5-R: CTCTGGGTTGGCACACACTT

Mm Ccl5-F: TTTGCCTACCTCTCCCTCG

Mm Ccl5-R: CGACTGCAAGATTGGAGCACT

Mm Slpi-F: GGCCTTTTACCTTTCACGGTG

Mm Slpi-R: TACGGCATTGTGGCTTCTCAA

Mm Mmp12-F: CTGCTCCCATGAATGACAGTG

Mm Mmp12-R: AGTTGCTTCTAGCCCAAAGAAC

Mm Ccl7-F: GCTGCTTTCAGCATCCAAGTG

Mm Ccl7-R: CCAGGGACACCGACTACTG

Mm Tnfr2-F: ACACCCTACAAACCGGAACC

Mm Tnfr2-R: AGCCTTCCTGTCATAGTATTCCT

Mm Grn-F: ATGTGGGTCCTGATGAGCTG

Mm Grn-R: GCTCGTTATTCTAGGCCATGTG

Hs Grn F: ATCTTTACCGTCTCAGGGACTT

Hs Grn R: CCATCGACCATAACACAGCAC

Hs TNFR2 F: CGGGCCAACATGCAAAAGTC

Hs TNFR2 R: CAGATGCGGTTCTGTTCCC

Hs CCL5 Sequencing F: TTAGGGGATGCCCCTCAACT

Hs CCL5 Sequencing R: CTGAGACTCACACGACTGCTG

Hs Rab27a-F: GCTTTGGGAGACTCTGGT

Hs Rab27a-R: TCAATGCCCACTGTTGTGATAAA

Mm Rab27a-F: TCGGATGGAGATTACGATTACCT

Mm Rab27a-R: TTTTCCCTGAAATCAATGCCCA

mCCL5i sgRNA targets

AGAGATCTTCATGGTACCCG

GTACCCGCGGCAGAGGCTGT

ATCTCCCACAGCCTCTGCCG

CTTCATGGTACCCGCGGCAG

GGCAGCTGCAGAGATCTTCA

mCCL5a sgRNA targets

TTATGACAGCAACAAGTGTT 
TGGAAACTCCCCAAGTCCTG GTGGAAACTCCCCAAGTCCT TGTGGAAACTCCCCAAGTCC CCCCCCAGCCCCAGGACTTG

\subsection{Antibodies, Cytokine Arrays, ELISAs}

RKIP (derived in lab from serum of rabbits exposed to an RKIP peptide), Rabbit antiCD63 antibody (System Biosciences, Palo Alto, CA, USA), Rab27a (AF7245, R\&D Systems, Minneapolis, MN, USA), F4/80 (MCA497GA, AbD Serotec, Bio-Rad, Hercules, CA, USA), Foxp3 clone:FJK-16s (13-5773, eBioscience, San Diego, CA, USA), FITC-CD63 (cat \# 550759, BD, Franklin Lakes, NJ, USA), CD9-PerCP-Cy 5.5 (561329, BD), CD81-Pacific Blue (349515, BD), Alix Ab (3A9) (65678, Novus, Centennial CO, USA), APC-Fire IgG (406623, BioLegend, San Diego, CA, USA), Exosome-human CD63 isolation/detection reagent (10606D, Life Technologies, Waltham, MA, USA), CCL5 ELISA (ELH-RANTES-1, Ray Biotech, Peachtree Corners, GA, USA), Mouse Cytokine Array (L308, Ray Biotech)

\subsection{Tumor Growth Assays}

Two thousand tumor cells were plated in $200 \mu \mathrm{L}$ of media per well in a 96-well culture plate (Falcon, Corning, NY, USA). Six replicates per condition were used for all assays. Cells were then monitored using an Incucyte Zoom (Essen Bioscience, Sartorius, Ann Arbor, MI, USA), with $20 \times$ phase-contrast images taken every four hours. Percent confluence was calculated using Incucyte Analysis Software and exported as a CSV file for either individual time and data points or average data points per time point. Exported data was graphed as individual points (when available) using Graph Pad Prism and statistical analysis performed as described below.

\subsection{Tumor Migration Assays}

20,000 tumor cells were plated in $200 \mu \mathrm{L}$ of media per well in a 96-well culture plate (Falcon) and allowed to adhere overnight. Six replicates per condition were used for all assays. Each well was precisely scratched using an Incucyte Woundmaker Tool (Essen Bioscience, Sartorius). Wells were washed to remove scratched cells and debris, and $200 \mu \mathrm{L}$ of fresh media per well was added. Cells were then monitored using an Incucyte Zoom (Essen Bioscience, Sartorius), with $20 \times$ phase-contrast images taken every four hours. Migration was calculated as (\%Wound Healing) using the Incucyte Scratch Wound Analysis Software Module and exported as a CSV file for either individual time and data points or average data points per time point. Exported data was graphed as individual points (when available) using Graph Pad Prism and statistical analysis performed as described below.

\subsection{Invasion Assays}

As previously described, $2 \times 10^{4} \mathrm{BM} 1$ cells or $1 \times 10^{5}$ E0771-LMB cells were plated in 24-well trans-well inserts with $8 \mu \mathrm{m}$ pores (Corning, 353097) coated with growth factor depleted basement membrane extract (Trevigen, Gaithersburg, MD, USA) [11,38]. After incubating at $37^{\circ} \mathrm{C}$ for $24 \mathrm{~h}$, inserts were transferred to an empty well and stained with $4 \mathrm{ng} / \mu \mathrm{L}$ of Calcein AM (Corning) for one hour. Stained cells were gently wiped with Q-tips to remove cells on the top layer of the insert, then placed in non-enzymatic dissociation solution (Trevigen) using gentle shaking for one hour at $37^{\circ} \mathrm{C}$ and $150 \mathrm{RMP}$. Fluorescence was measured using a Victor X3 fluorescent plate reader with excitation at $465 \mathrm{~nm}$ and emission at $535 \mathrm{~nm}$.

\subsection{Tumor EV Isolations}

Tumor EV isolations were done in accordance with MISEV2018 guidelines [39]. For all experiments, tumor conditioned media (CM) was spun at $2000 \times g$ for $10 \mathrm{~min}$ to remove cells and cell debris, and then filtered through a $0.22 \mu \mathrm{m}$ PES syringe filter (Millipore). For ultracentrifugation experiments, prepared CM was spun at $100,000 \times g$ for $70 \mathrm{~min}$ 
in polycarbonate, hard-wall tubes. EV cleared CM was then removed and saved for experiments. Remaining media was removed and EV pellets were resuspended in the same starting volume of serum free media from which they were enriched.

For Izon qEV columns CM from four 15-cm plates was prepared as above, followed by concentration using an Amicon with a $3 \mathrm{kD}$ pore (MilliporeSigma, Darmstadt, Germany) to $500 \mu \mathrm{L}$. Concentrated media was loaded onto a rinsed qEV column. Fractions 1-6 (column dead volume, $3 \mathrm{~mL}$ ) were collected in one tube, followed by collection of EVs in fractions 7-9 (1.5 mL). Following isolation, columns were cleaned with $750 \mu \mathrm{L}$ of $0.5 \mathrm{M} \mathrm{NaOH}$ followed by washing with $30 \mathrm{~mL}$ of PBS (Corning) before re-use.

\subsection{SEM Measurements of EVS}

Copper grids were glow discharged for $30 \mathrm{~s}$, stained with $3.5 \mu \mathrm{L}$ of sample for $1 \mathrm{~m}$, rinsed twice with 1\% PTA, and then stained with 1\% PTA for 45s. Grids were imaged on an FEI Technai G2 F30 300kV transmission electron microscope.

\subsection{Nano Tracking Analysis (NTA) Utilizing the Malvern NS300}

Enriched EV samples were diluted 1:20 in PBS. $1 \mathrm{~mL}$ of diluted EVs were then used for NTA. Once the flow cell and tubing were primed with the sample, the syringe pump was set to a flow rate of $50 \mu \mathrm{L} / \mathrm{s}$. Three videos of $45 \mathrm{~s}$ were recorded of enriched EVs. The videos were processed according to standard Malvern protocols and then exported.

\subsection{Flow Cytometry}

We collected conditioned media from cells plated at $1 \times 10^{6}$ cells and EVs were isolated as described above using $\mathrm{qEV}$ original. Next, we isolated exosomes from other EVs using a human CD63 Dynabeads (Invitrogen, Waltham, MA, USA), per the company's protocol for use in flow cytometry. The exosomes conjugated with Dynabeads were washed in $300 \mu \mathrm{L}$ of Dynabead's isolation buffer. One hundred microliters of exosomes were stained in separate polystyrene tubes with $0.1-10 \mu \mathrm{g} / \mathrm{mL}$ of Alix1, CD9, or CD63 (each antibody was measured individually) and were incubated for $60 \mathrm{~min}$ at room temperature. The exosomes were washed three times using $300 \mu \mathrm{L}$ of isolation buffer and then placed on magnetic stand and discard the supernatant. This step was repeated once and resuspended in $500 \mu \mathrm{L}$ of PBS with $0.02 \%$ sodium azide. The exosomes were kept in foil and immediately analyzed using a BD Fortessa 4-15. Each antibody was measured independently, so no compensation was needed. Unstained and isotype controls were measured to establish a baseline for gating negative particles. Analysis of flow data and graphs were produced using FlowJo.

\subsection{Tumor Educated Macrophage Programming}

Bone marrow was isolated from the femur and tibia of 6-10-week-old C57Bl/6 mice (Charles Rivers). Red cell lysis buffer (Santa Cruz) was used to remove red blood cells. Remaining bone marrow was counted, and 1 million cells were plated per well in a 6-well plate and cultured in 70\% RPMI 1640 supplemented with 10\% FBS (Corning) and 50 U/mL penicillin, and $50 \mu \mathrm{g} / \mathrm{mL}$ streptomycin (Invitrogen) mixed with 30\% L929 Conditioned Media. Media was replenished at days two and six during culture. On day seven, bonemarrow derived macrophages (BMDMs) were washed 2-3 times with PBS (Corning) and then treated with $20 \mathrm{ng} / \mathrm{mL}$ of mouse M-CSF spiked into tumor cell conditioned media, serum free media containing tumor EVs, or serum free media as a negative control. EV size and numbers were determined after isolation using nanoSight. $1 \times 10^{8} \mathrm{EVs} / \mathrm{mL}$ were used for programming with BM1 tumor EVs or $1 \times 10^{9} \mathrm{EVs} / \mathrm{mL}$ for LMB EVs.

For TEMs, after programming, cells were washed with PBS 2-3 times. Then $1 \mathrm{~mL}$ of serum free DMEM was added. After incubation at $37^{\circ} \mathrm{C}$ for $24 \mathrm{~h}$, conditioned media was removed and cells and cell debris were removed by centrifugation at $2000 \times g$ for $10 \mathrm{~min}$, and immediately used to treat TNBC cells. TEMs were lysed with TRIzol and RNA extracted using Direct-zol kit (Zymo Research, Irvine, CA, USA) per manufacturers protocol. 


\subsection{Tumor Associated Macrophage Isolations}

As previously described [11], tumors were grown to approximately $0.75 \mathrm{~g}$ before being harvested. Tumors were dissociated both physically with scissors to 1-2 $\mathrm{mm}$ pieces and using C-tubes and a gentleMACS dissociator (Miltenyi Biotech, Bergisch Gladbach, Germany) as well as enzymatically using the human tumor dissociation kit (Miltenyi Biotech). Cells were filtered through a $70 \mu \mathrm{m}$ mesh filter. Mononuclear cells were isolated using Ficoll-Paque PREMIUM (GE Healthcare, Chicago, IL, USA) gradient centrifugation at 420 RPM for $45 \mathrm{~min}$. Macrophages were then obtained using CD11b positive selection beads (Miltenyi Biotech).

For tumor derived macrophages, $1 \times 10^{6}$ TAMs were plated in one well of a 6-well plate. After $30 \mathrm{~min}$, cells were washed with PBS to ensure only viable macrophages attached to the plate remained. Cells were incubated for $24 \mathrm{~h}$ to obtain conditioned media in serum free DMEM. Cells and cell debris were removed by centrifugation at $2000 \times g$ for $10 \mathrm{~min}$ prior to use in subsequent assays.

\subsection{Conditioned Media}

For TEM conditioned media, cells were washed twice with PBS and once with serum free DMEM after $48 \mathrm{~h}$ of programming with CM or EVs. Each well was then incubated in serum free DMEM for $24 \mathrm{~h}$ to collect TEM CM. TEM CM was then spun at $2000 \times g$ for $10 \mathrm{~min}$ to remove cells and cell debris.

For tumor derived macrophages, $5 \times 10^{5}$ TAMs were plated in one well of a 6-well plate. After $30 \mathrm{~min}$, cells were washed with PBS to ensure only viable macrophages attached to the plate remained. Cells were incubated for $24 \mathrm{~h}$ to obtain conditioned media in serum free DMEM. Cells and cell debris were removed by centrifugation at $2000 \times g$ for $10 \mathrm{~min}$.

\subsection{Mice}

All mice were housed and handled according to the University of Chicago Institutional Animal Care and Use Committee guidelines. Athymic nude, Balb/c mice, and C57Bl/ 6 were purchased from Charles Rivers. Mice were injected with $2 \times 10^{6}$ human or $5 \times 10^{5}$ mouse tumor cells in $100 \mu \mathrm{L}$ of DPBS into the fat pad of 5-6-week-old female mice. Tumor volumes were measured twice per week and calculated as volume $=(\pi / 6) \times$ width $^{2} \times$ length, where width is the shorter of the two distances. Tumors were grown to $\sim 1000 \mathrm{~cm}^{3}$ (or $\sim 0.75-1 \mathrm{~g}$ ) and removed for analysis.

For LMB co-injection studies, TEMs were injected into the fat pad of C57Bl/ 6 mice mixed with E0771-LMB cells at a ratio of 1 TEM:1 tumor cell. Lung metastases were assayed at the end of the study by fixing in formalin and sectioning. Six $5 \mu \mathrm{m}$ sections were quantified for number and size of metastases. Sections were $100 \mu \mathrm{m}$ apart, and number of metastases counted were added to give number of metastases per lung.

For BM1 co-injection studies, TAMs isolated from BM1 tumors in mice were immediately injected into the fat pad of nude mice mixed with MDA-MB-231 tumor cells at a ratio of 1 TAM: 2 Tumor cells.

\subsection{Immunohistochemistry}

All immunohistochemistry was performed at the University of Chicago Human Tissue Resource Center. Tissue sections were deparaffinized and rehydrated through xylenes and serial dilutions of $\mathrm{EtOH}$ to deionized water. For F4/80, samples were incubated in antigen retrieval buffer (S1699, DAKO, Agilent, Santa Clara, CA, USA) and heated in a steamer at $97^{\circ} \mathrm{C}$ for $20 \mathrm{~min}$. Anti-mouse F4/80 antibody (1:200, MCA497GA, AbD Serotec) was applied on tissue sections for $1 \mathrm{~h}$ at room temperature. Following staining and TBS wash, tissue sections were incubated with biotinylated anti-rat IgG $(10 \mu \mathrm{g} / \mathrm{mL}, \mathrm{BA}-4001$, Vector laboratories) for $30 \mathrm{~min}$ at room temperature. The antigen-antibody binding was detected by Elite kit (PK-6100, Vector Laboratories) and DAB (DAKO, K3468) system according to the manufacturers' protocols. Following staining, tissue sections were briefly immersed in hematoxylin for counterstaining and were covered with cover glasses. Stained 
tissue sections were scanned at $20 \times$ magnification and analyzed using Aperio Imagescope ePathology software. To quantify infiltrating macrophages, number of F4/80 positive cells were counted using Imagescope and normalized to the area imaged.

\subsection{TCGA Analysis}

All analysis of human tumor samples from the cancer genome atlas (TCGA) were done using cbioportal.org. $z$-score normalized mRNA expression was compared for the 1100 samples from the Breast Invasive Carcinoma (TCGA, Firehose Legacy) patient set with mRNA expression from RNAseq. Heatmaps and clinical data were ordered using heatmap clustering order (hierarchical clustering done by cBioPortal) and exported.

\subsection{Statistical Analysis}

All graphics were made, and all statistical analysis was done using Graph Pad Prism. Unless otherwise noted, bar graphs represent the mean \pm standard error of the mean (SEM). For comparing statistical differences between means of multiple samples a standard two-way ANOVA was used. For comparisons between paired groups, a standard two-way ANOVA was used. For all comparisons, p-values were corrected for multiple comparisons using Tukey's multiple comparisons test. Correlations were done using a Spearman correlation. Statistical tests used are noted in figure legends.

\section{Results}

\subsection{Tumor EVs Program Macrophages to a Pro-Metastatic Phenotype In Vitro and In Vivo}

To investigate whether tumor EVs educate macrophages toward a pro-metastatic TAM phenotype, we treated bone marrow-derived macrophages (BMDMs) with tumor EVs for $48 \mathrm{~h}$ in the presence of macrophage colony stimulating factor (M-CSF), creating tumor EVeducated macrophages (TEMs) (Figure 1A, Figures S1 and S2; Appendix A). Isolated EVs were characterized per MISEV2018 guidelines (Figure S2A,B) [39]. Macrophages treated with tumor EVs drove tumor cell invasion at a higher rate than the untreated control (Figure 1B). Tumor EVs induced expression in macrophages of pro-invasive markers Ccl7, Tnfr2, Mmp12, and Slpi compared to an M-CSF only control (Figure 1C) [11].

To determine whether tumor EV secretion plays an active role in macrophage-induced tumor cell invasion, we knocked down Rab27a, a known regulator of the EV secretion machinery (Figure S3A) [22,26]. Expression of shRNAs for Rab27a in human BM1 and mouse LMB tumor cells decreased EV secretion by 50\% (measured by NTA), similar to previous reports [22,40] (Figure S3B-I). Considering that the Rab family of proteins are also regulators of endosomal sorting and membrane fusion, we also investigated if knockdown of Rab27a might affect other relevant cell properties. However, we observed no change in tumor cell growth, migration, or invasion of shRab27a cells relative to controls (Figure S4). Next, we educated macrophages using EVs from an equal number of control or Rab27a-knockdown tumor cells to generate TEMs, allowing differences in EV secretion to affect macrophage education. The TEMs from these two conditions were then used in our invasion assay (Figure 1A). We observed significantly reduced tumor cell invasion when TEMs were educated using EVs from either shRab27a BM1 and shRab27a LMB cells compared to controls (Figure 1D). Consistent with this result, tumor media depleted of EVs by ultracentrifugation was unable to program macrophages capable of driving tumor cell invasion (Figure 1E). Together, these results suggest that tumor EVs are a necessary component for programming macrophages to promote a pro-metastatic phenotype in vitro.

To determine if reducing tumor EV secretion impacts in vivo macrophage polarization and metastasis, we injected BM1 tumor cells (+/ - shRab27a) into the inguinal fat pads of athymic nude mice (with an intact innate immune system [41,42]). We utilized shRab27a (clone sh-5) as it produced the most robust change in EV secretion and in vitro macrophage programming. After tumors were established in the mice, we measured tumor size, tumor cells circulating in the blood stream, and macrophages recruited to tumors. Reducing EV secretion through Rab27a knockdown did not affect the size of BM1 tumors (Figure 2A). 
However, fewer tumor cells were found in circulation in the shRab27a group compared to control (Figure 2B). This is consistent with a role of tumor EVs in the early stages of metastasis (tumor cell intravasation into the bloodstream). As previously noted, shRab27a did not affect cell autonomous invasion nor migration (Figure S4B,C), suggesting that the effects on tumor intravasation are due to changes in cells within the tumor microenvironment, not the inherent invasive ability of the tumor cells. We therefore wanted to explore whether reducing tumor EV secretion affected the recruitment of TAMs. When we stained for F4/80, a surface glycoprotein frequently found on mouse macrophages, we found no significant difference in the number of TAMs recruited to control versus shRab27a tumors (Figure 2C,D).

A

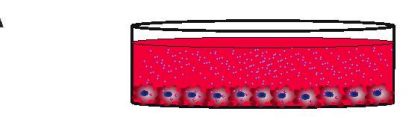

Tumor EV-Educated Macrophages (TEMs)

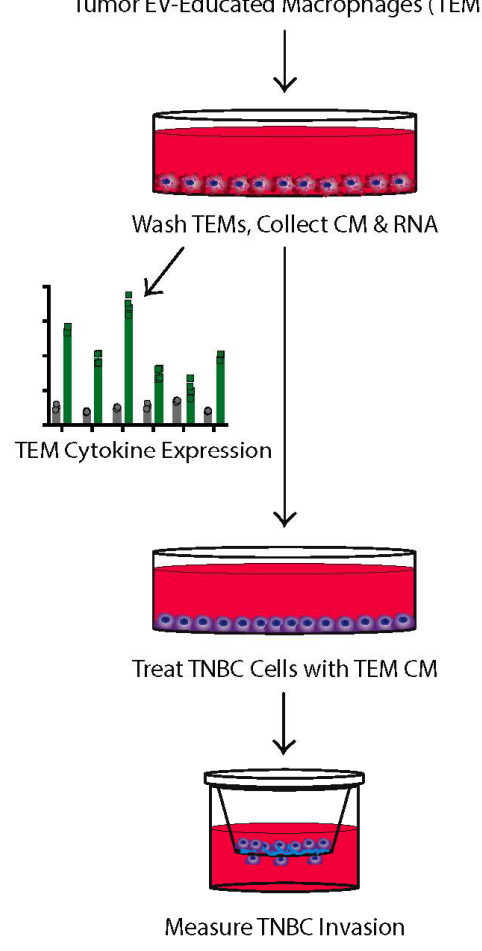

B
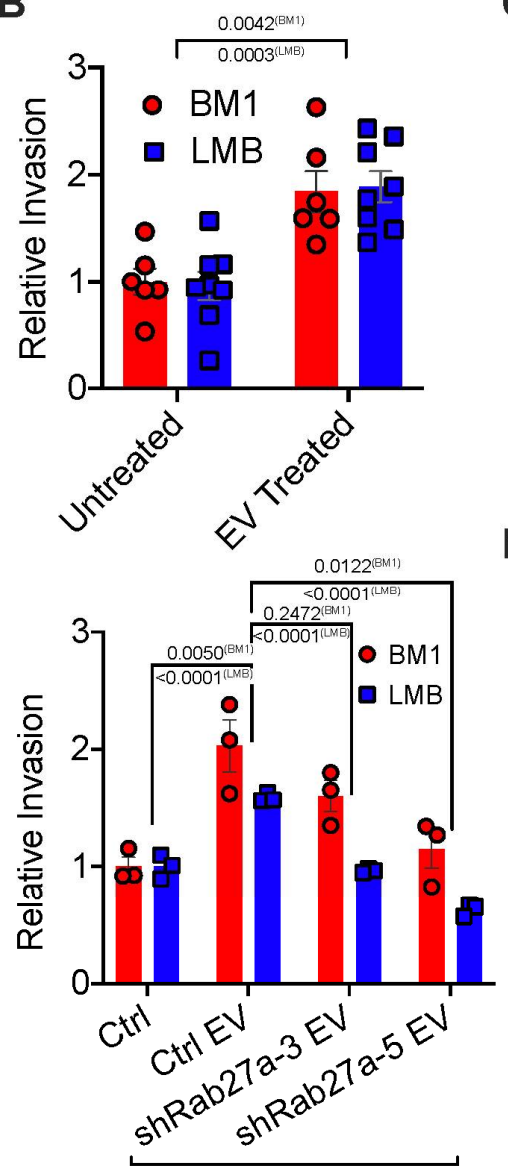

C
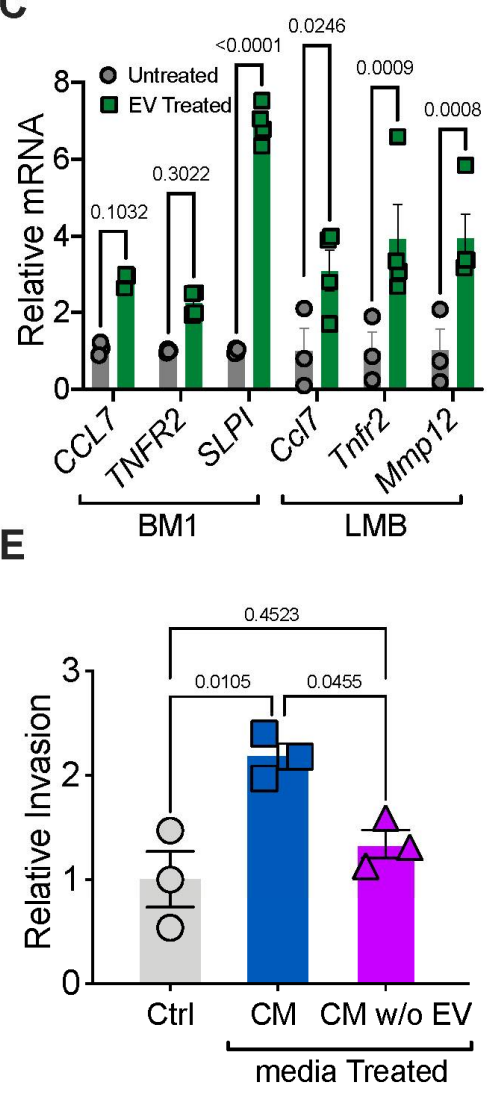

EV Treated

Figure 1. Tumor EVs are required to program macrophages to a pro-metastatic phenotype in vitro (A) Schematic describing the method by which TEMs were programmed with tumor EVs, followed by analysis of TEM gene expression and effect of TEMs to TNBC invasion. TNBC Cells BM1 and LMB were used for all experiments. P-values were calculated using a two-way $t$-test $(\mathbf{B})$ Relative invasion of TNBC cells pre-incubated for $24 \mathrm{~h}$ with TEM conditioned media $(N=6)$. $($ C) qRT-PCR of Ccl7, Tnfr2, Slpi, and Mmp12 in TEMs programmed with TNBC EVs compared to M-CSF alone (Control) with Gapdh as a loading control. P-values were calculated using a two-way ANOVA ( $N=3$ for LMB; $N=4$ for BM1). (D) Relative invasion of TNBC cells pre-incubated for $24 \mathrm{~h}$ with TEM conditioned media $(N=3)$. TEMs were programmed with M-CSF (Control) or EVs from control (Ctrl EV) or sh-Rab27a tumor cells (shRab27a clones: sh3, sh5). P-values were calculated using a one-way ANOVA comparing changes in BM1 and LMB cells separately between groups (E) Relative BM1 invasion (mean +/ - SEM) when cells were treated with TEM media programmed by an M-CSF control (control, grey), BM1 media with EVs (CM, red), or BM1 media with EVs removed (CM w/o EV, purple). p-values were calculated using a one-way ANOVA comparing both changes in both BM1 and LMB cells together between groups. 
A

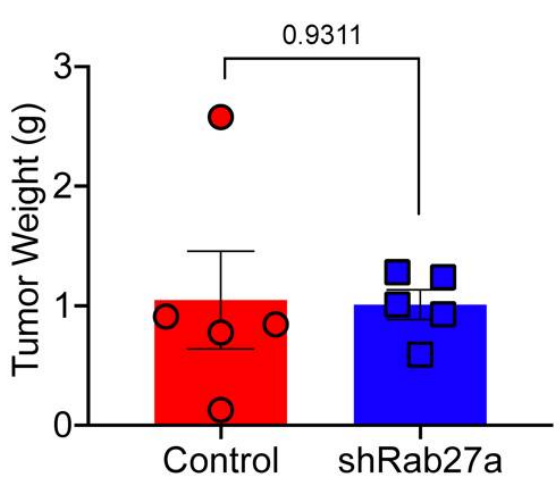

C

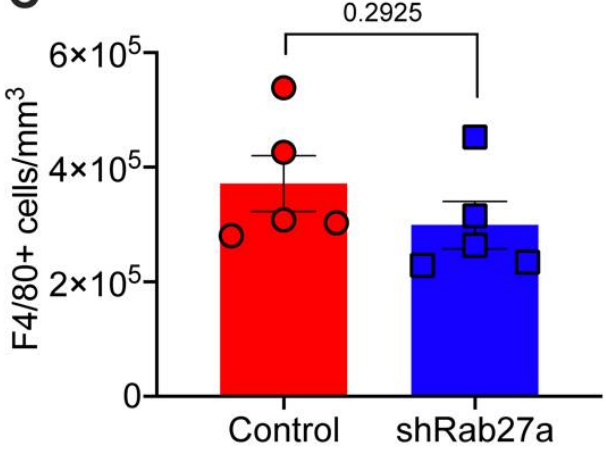

B

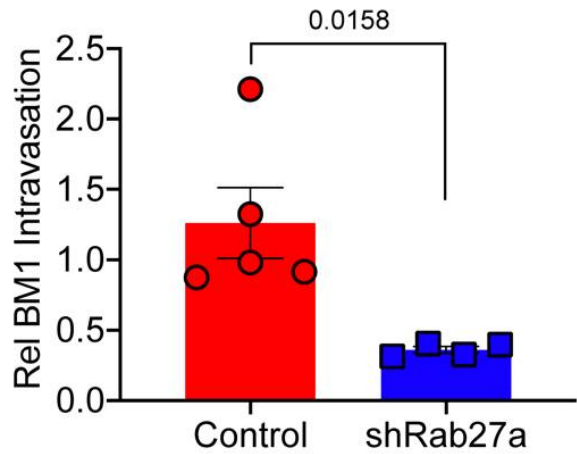

D

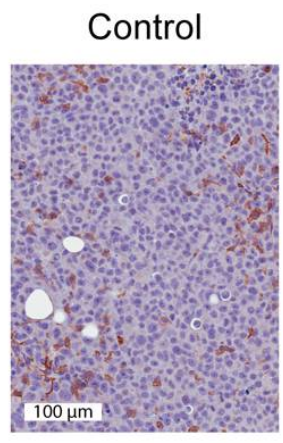

shRab27a

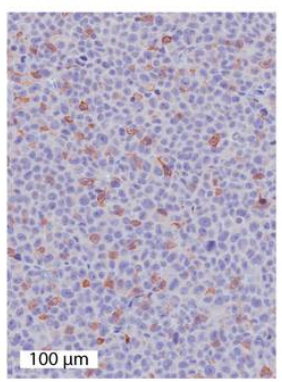

Figure 2. Tumor EVs generate TAMs that promote tumor intravasation in vivo (A-D) BM1+Control vector (Control, Red) or BM1+shRab27a (sh-5) (shRab27a, Blue) cells $\left(2 \times 10^{6}\right)$ were injected into athymic nude mice. (A) Final tumor weights at sacrifice $(N=5)$. (B) Relative intravasation of tumor cells into the blood stream measured by quantifying the ratio of human GAPDH (tumor) to mouse Gapdh (white blood cells) by qRT-PCR $(N=5)$ from blood taken immediately prior to sacrifice. (C) Macrophage infiltration was quantified as the number of positive cells normalized to area using Aperio. ( $N=5$ per group). (D) Representative images of F4/80-stained tumor sections in Control and shRab27a tumors. P-values between two groups were calculated using a $t$-test.

Because we saw no differences in macrophage number recruited to tumors when tumor Rab27a expression was reduced, we next sought to determine how EV education of macrophages alters their phenotype in vivo. We isolated macrophages from control and shRab27a BM1 tumors (Figure 2D). We then collected conditioned media from the isolated macrophages and tested the ability of secreted factors in the media from these macrophages to drive tumor cell invasion. We found that macrophages from shRab27a tumors failed to drive tumor cell invasion at the same rate as macrophages from control tumors (Figure 3A). Because this was the same phenotype observed in the in vitro programmed macrophages, we decided to compare how shRab27a altered macrophage biology in vitro to in vivo. To test this, we compared differential expression of cytokines in TEMs (in vitro) to TAMs (in vivo) upon reduction of EV secretion from tumor cells by shRab27a. We observed that $\sim 30 \%$ of differentially expressed proteins overlapped between our in vitro TEM and in vivo TAM polarization experiments. These included CCL7, CCL19, CXCL7, NRG3, OPN, HGF, and TGFB3 (Figure 3B, Figure S5A-C), known drivers of immune evasion and metastasis $[11,43,44]$. These findings provide further support that tumor EVs are an essential component for programming macrophages toward a pro-metastatic TAM phenotype in the tumor microenvironment. Thus, reducing tumor EV secretion sufficiently decreases both macrophage cytokine secretion and macrophage-driven tumor cell invasion (Figure 3C). 
A

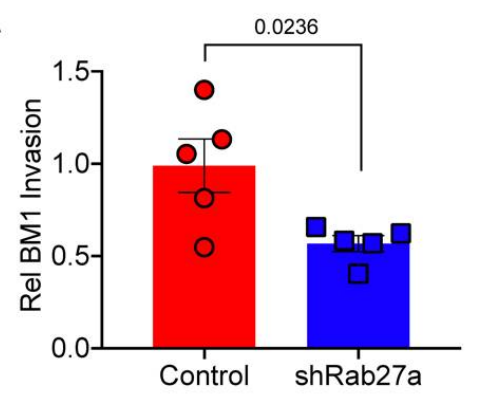

B

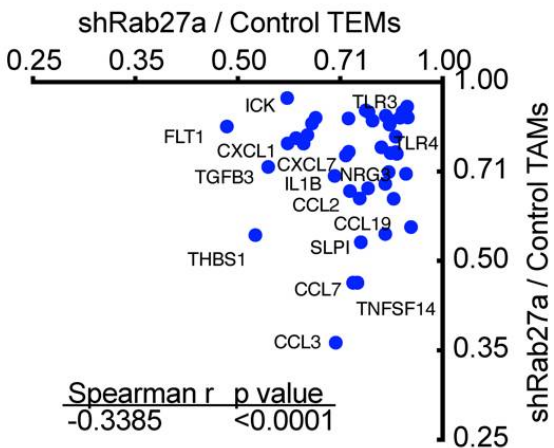

C
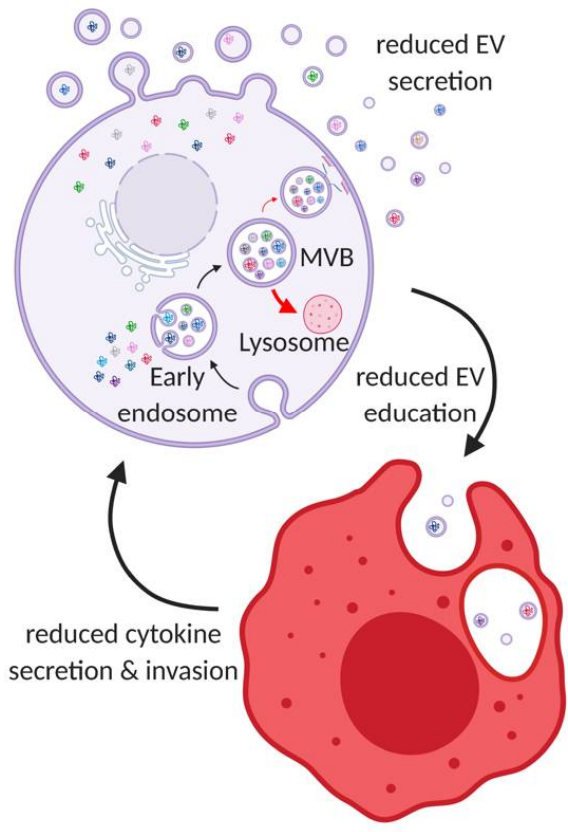

Figure 3. Tumor EVs regulate macrophage phenotype in vivo (A) Invasion assay of BM1 cells treated with $\mathrm{CM}$ of TAMs derived from control or shRab27a expressing BM1 tumors $(N=5)$. ( $p$-values were calculated using a one-way ANOVA). (B) Spearman correlations of macrophages proteins assayed using an L-308 Cytokine Array ( $N=3$ mice (TEMs) or 5 mice (TAMs), pooled for array analysis). Zoom-in of the lower left quadrant of a Spearman correlation of cytokines regulate by shRab27a EV secretion in TEMs vs TAMs (showing the 42 proteins reduced by shRab27a in both TEMs and TAMs). X-axis: comparison of proteins in shRab27a to Control TEMs. $Y$-axis: comparison of proteins in shRAb27a versus Control TAMs. Correlation determined using a Spearman test. (C) Schematic summarizing Rab27a-dependent EV secretion and subsequently macrophage programming. Resulting EV programmed macrophages drive increase tumor cell invasion. Created using Biorender.com (accessed on 2 June 2021).

\subsection{Tumor CCL5 Expression Regulates EV Secretion and EV Education of Macrophages}

To explore the tumor intrinsic mechanism responsible for tumor EV-driven macrophage education, we investigated whether tumor cytokines that recruit macrophages also participate in macrophage education to promote metastasis. We focused on the potential role of CCL5, a chemokine that we and others previously identified to be a key mediator of macrophage recruitment in TNBC $[10,11]$. First, we tested if direct CCL5 stimulation of macrophages was sufficient to drive them toward a pro-tumor phenotype. Indeed, neither M-CSF nor M-CSF+CCL5 stimulation of macrophages promoted tumor cell invasion (Figure S6A). Additionally, neither treatment induced expression of pro-metastatic markers Ccl7, Mmp12, and Grn (Figure S6B). However, direct stimulation of tumor cells with CCL5 led to an increased macrophages capable of driving tumor cell invasion (Figure S6C). We hypothesized that tumor CCL5 expression regulates macrophage reprogramming through an indirect mechanism. We first tested how increased tumor CCL5 expression regulated tumor EV secretion. When CCL5 was overexpressed (CCL5 $\left.{ }^{\text {high }}\right)$ in human BM1 or mouse LMB TNBC cells (Figure 4A), we observed a slight but not significant increase in EV secretion from comparable tumor cell numbers (Figure 4B and Figure S6). Conversely, reduction of CCL5 expression through either CRISPR-Cas9 (BM1 cells) or CRISPR-dCas9-KRAB (LMB cells) (Figure 4C) decreased tumor EV secretion with one guide RNA (Figure 4D and Figure S7). When we examined EVs from CRISPR-dCas9-KRAB KD cells, we found a significant reduction in $C c l 5$ copies present (Figure S7I). 
A

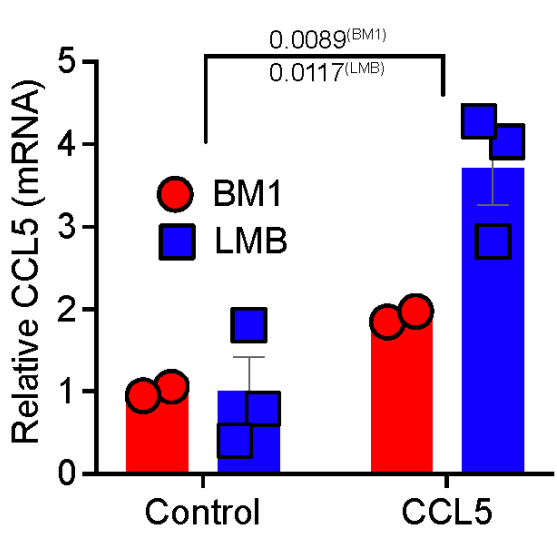

C

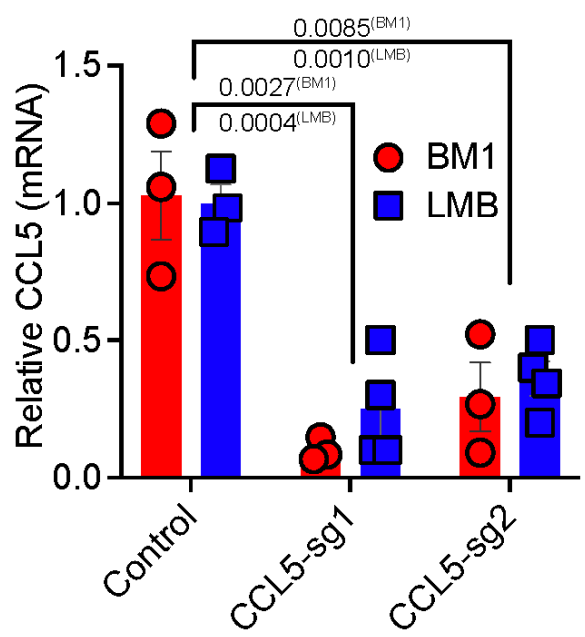

B

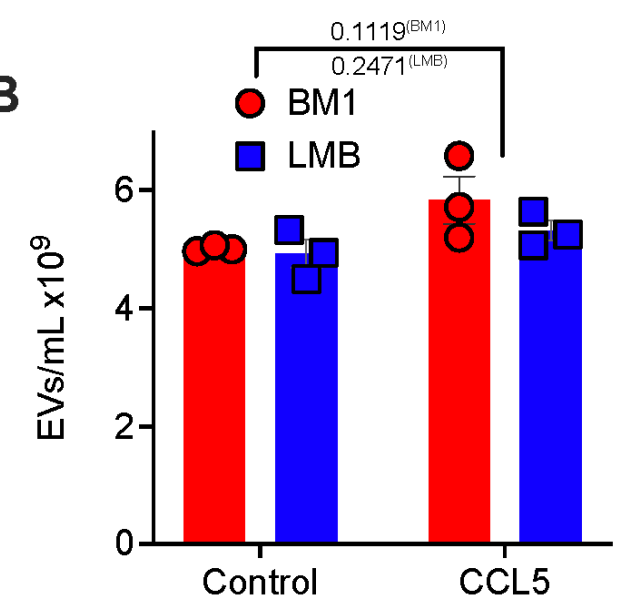

D

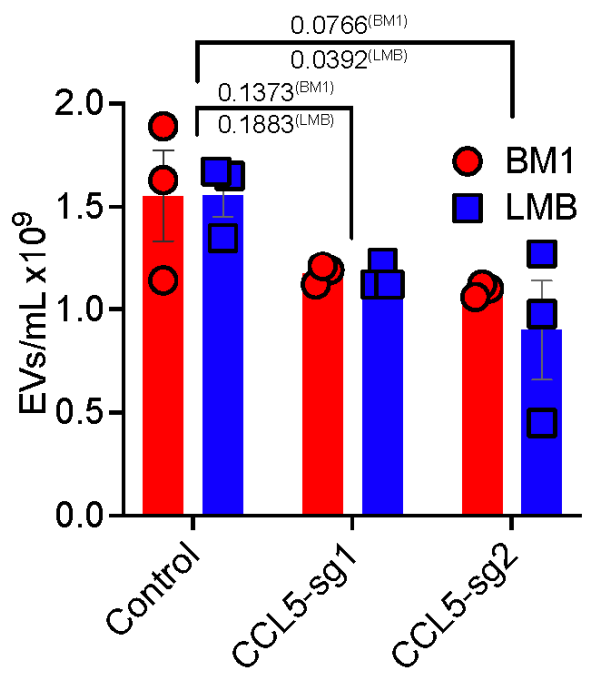

Figure 4. Tumor CCL5 expression regulates EV secretion (A) qRT-PCR of CCL5 in BM1 using GAPDH as loading control or ddPCR of Ccl5 in LMB cells. Relative expression of CCL5/Ccl5 in CCL5 overexpressing cells (CCL5) is compared to those with a control vector (Control). $p$-value was determined using a two-tailed $t$-test $(N=3)$. (B) Number of EVs/mL determined by NTA analysis using a Nanosight in BM1 and LMB cells either expressing a control vector (control) or CCL5 over-expressing (CCL5). $p$-value was determined using a two-tailed $t$-test $(N=3)$. (C) qRT-PCR of CCL5 in BM1 using GAPDH as loading control or ddPCR of Ccl5 in LMB cells. Relative expression of CCL5/Ccl5 in BM1 and LMB cells using CRISPR/Cas9 KO (BM1) or CRISPR/dCas9-KRAB (LMB). CCL5 reduced cells (sg-1, sg-2) are compared to those with a control vector (Control). $p$-value was determined using a one-way ANOVA $(N=3)$. (D) Number of EVs/mL determined by NTA analysis using a Nanosight in BM1 and LMB cells either expressing a control vector (control) or CRISPR/Cas9 KO (BM1), CRISPR/dCas9-KRAB (LMB) with a CCL5/Ccl5 targeting guide RNA (sg-1, sg-2). p-values were determined using a one-way $\operatorname{ANOVA}(N=3)$.

Knowing that CCL5 regulates the number of EVs that tumor cells secrete, we also wanted to test if it regulates the cargo of the EVs and subsequently their impact on macrophages. When we treated macrophages with the same number of control and CCL5 $5^{\text {high }}$ tumor EVs (quantitated by NTA), there was an approximately $50 \%$ increase in TEM-driven tumor cell invasion (Figure 5A). These data clearly indicate that the cargo in $\mathrm{EVs}$, and not the number of EVs, drive functional programming of macrophage to drive tumor cell invasion. Conversely, reduction of CCL5 expression through either CRISPR-Cas9 (BM1 cells) or CRISPR-dCas9-KRAB (LMB cells) (Figure 4C) decreased TEM-mediated tumor cell invasion (Figure 5B). To determine the effect of CCL5 on macrophage reprogramming, we analyzed cytokines in the conditioned media from TEM or TAM using an L308 
mouse cytokine array (Figure S8). We generated TEMs in vitro using EVs from sgCCL5 or control TNBC cells, and identified TEM cytokines whose expression was decreased upon loss of tumor cell CCL5 expression (Figure S8A). We then compared them to cytokines in TAMs from CCL5 ${ }^{\text {high }}$ in vivo tumors [11] (Figure 5C and Figure S8B). Interestingly, CCL7, CCL19, CXCL7, NRG3, SLPI, OPN, and HGF in macrophages are upregulated in response to increased tumor CCL5 expression (Figure 5C). Overall, these experiments suggest that tumor CCL5 not only enhances the number of EVs but also alters the cargo of EVs. Analysis of these differentially expressed cytokines indicated that pro-metastatic osteopontin (OPN) [44] and SLPI [45-48] in both TEMs (in vitro) and TAMs (in vivo) are most strongly induced by tumor CCL5. Our previous work had established OPN and SLPI as markers of macrophages activated by CCL5 [11], and both of these factors have been shown to directly regulate tumor cell invasiveness [44-48]. These data represent a positive feedback loop between tumor and macrophage mediated by CCL5 and EVs (Figure 5D).

\subsection{Metastatic Tumor EVs Educate Macrophages to Promote Tumor Metastasis In Vivo}

To assess whether in vitro-generated TEMs are sufficient to promote metastasis in a syngeneic mouse model, we co-injected mouse TNBC cells (LMB) and LMB EV-educated macrophages (TEMs) into the inguinal fat pad of C57Bl/ 6 mice (Figure 6A). Co-injected TEMs had no effect on LMB tumor size (Figure 6B) but did increase both number and size of lung metastases compared to LMB tumor cells injected alone (Figure 6C,D). We observed a similar enhancement of the metastatic phenotype when we co-injected human BM1 tumor cells with in vivo-generated TAMs isolated from CCL $5^{\text {high }}$ BM1 tumors (Figure S9).

To determine whether the metastatic state of the tumor cells influences the ability of the educated macrophages to promote metastasis, we evaluated non-metastatic cells as a source of EVs (Figure S10, Appendix B). To generate a non-metastatic cell line for TNBC, we first expressed the metastasis suppressor, RKIP, [11] in LMB cells (Figure S10, Appendix B). We then used these RKIP-expressing LMB cells to produce EVs to program macrophages in vitro. In this case, co-injection of LMB cells with TEMs generated by these non-invasive, non-metastatic tumor EVs (RKIP+ LMB EVs) showed no significant change in lung metastases or tumor size compared to the TEM (-) control (Figure 6). These results suggest that in vitro EV-programmed TEMs phenocopy the metastatic state of the parental tumor cells from which the EVs are derived.

Although co-injection of TNBC tumor cells with in vitro-generated TEMs altered the function of tumor associated macrophages, recruitment of macrophages to the tumor did not change. When we stained for the macrophage marker F4/80, we found that the total number of macrophages in the tumor were the same between control tumors and those co-injected with TEMs (Figure 6E,G). This result suggests that the phenotype of the EV-educated macrophages, not simply the presence of macrophages alone, is driving increased metastasis.

Because EV programming of macrophages resulted in the secretion of cytokines known to recruit T-regs (CXCL1, CCL5, CCL19), we also immunostained tumors for the T-reg marker Foxp3. Co-injection of metastatic tumor cells (LMB) with TEMs generated by metastatic tumor EVs significantly increased the number of Foxp3+ T-regs compared to control (Figure 6F,H). Conversely, co-injection of metastatic tumor cells (LMB) with TEMs generated using non-metastatic tumor EVs (LMB+RKIP EVs) showed no change in T-reg recruitment (Figure $6 \mathrm{~F}, \mathrm{H}$ ). This result indicates that EVs, as a key regulator of macrophage phenotype, also indirectly affect the tumor microenvironment in vivo, driving it toward an immunosuppressive phenotype by altering macrophage biology to recruit T-regs. 
A

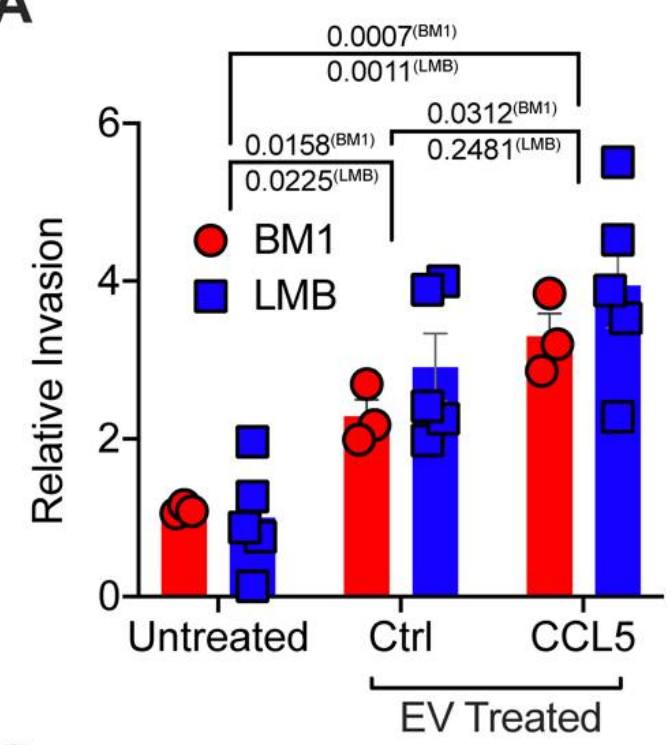

C

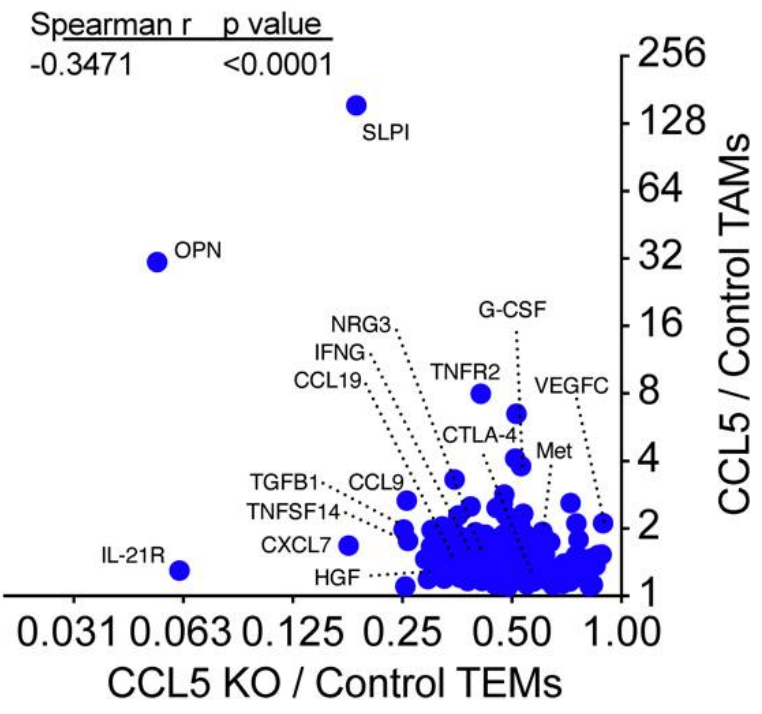

B

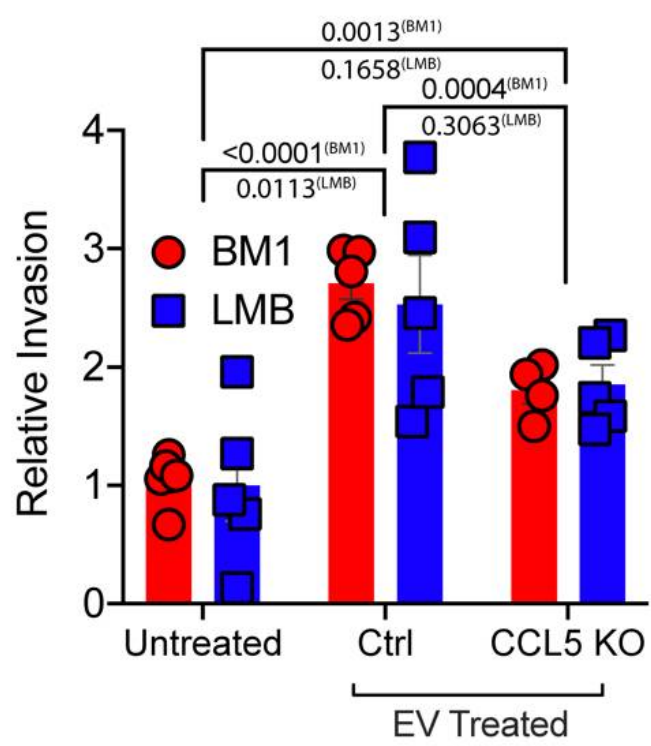

D

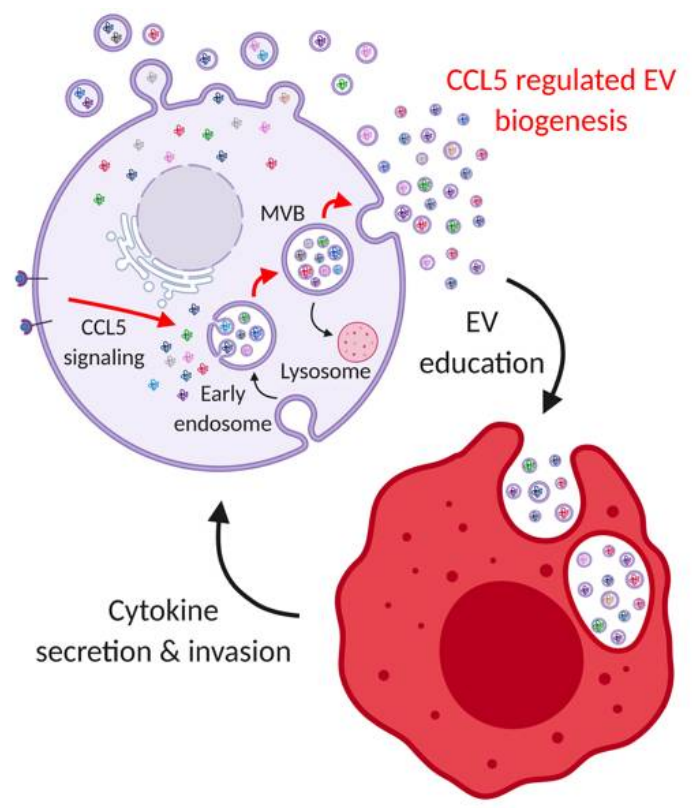

Figure 5. Tumor CCL5 expression regulates EV programming of macrophages. (A) Relative invasion of BM1 and LMB cells treated with TEM conditioned media (CM). TEMs were programmed with CSF-1 alone (Untreated), EVs from tumor cells with control vector (Ctrl), or EVs from tumor cells over-expressing CCL5 (CCL5). $p$-value was obtained using a one-way ANOVA comparing changes in invasion in each cell line upon TEM CM stimulation ( $N=3 \mathrm{BM} 1, N=5 \mathrm{LMB})$. (B) Relative invasion of BM1 and LMB cells treated with TEM conditioned media (CM). TEMs were programmed with CSF-1 alone (Untreated), EVs from tumor cells with control vector (Ctrl), or EVs from tumor cells with reduced CCL5 through CRISPR (CCL5 KO). $p$-value was obtained using a one-way ANOVA comparing changes in invasion in each cell line upon TEM CM stimulation $(N=5)$. (C) Spearman correlations of macrophages proteins assayed using an L-308 Cytokine Array $(N=3$ mice (TEMs) or 5 mice (TAMs), pooled for array analysis). Zoom-in of the upper left quadrant of a Spearman correlation of cytokines regulated by CCL5 in TEMs vs TAMs. Graph shows relative expression of proteins in BM1 CCL5 KO TEMs to BM1 Control TEMs. Y-axis: relative expression of proteins in BM1+CCL5 TAMs compared to BM1 Control TAMs. CCL5 TAM data set was used from previous publication for this analysis [11]. (D) Schematic summarizing CCL5 regulation of EV secretion and subsequently macrophage programming. Resulting CCL5-EV programmed macrophages drive increase tumor cell invasion. Created using Biorender.com (accessed on 2 June 2021). 


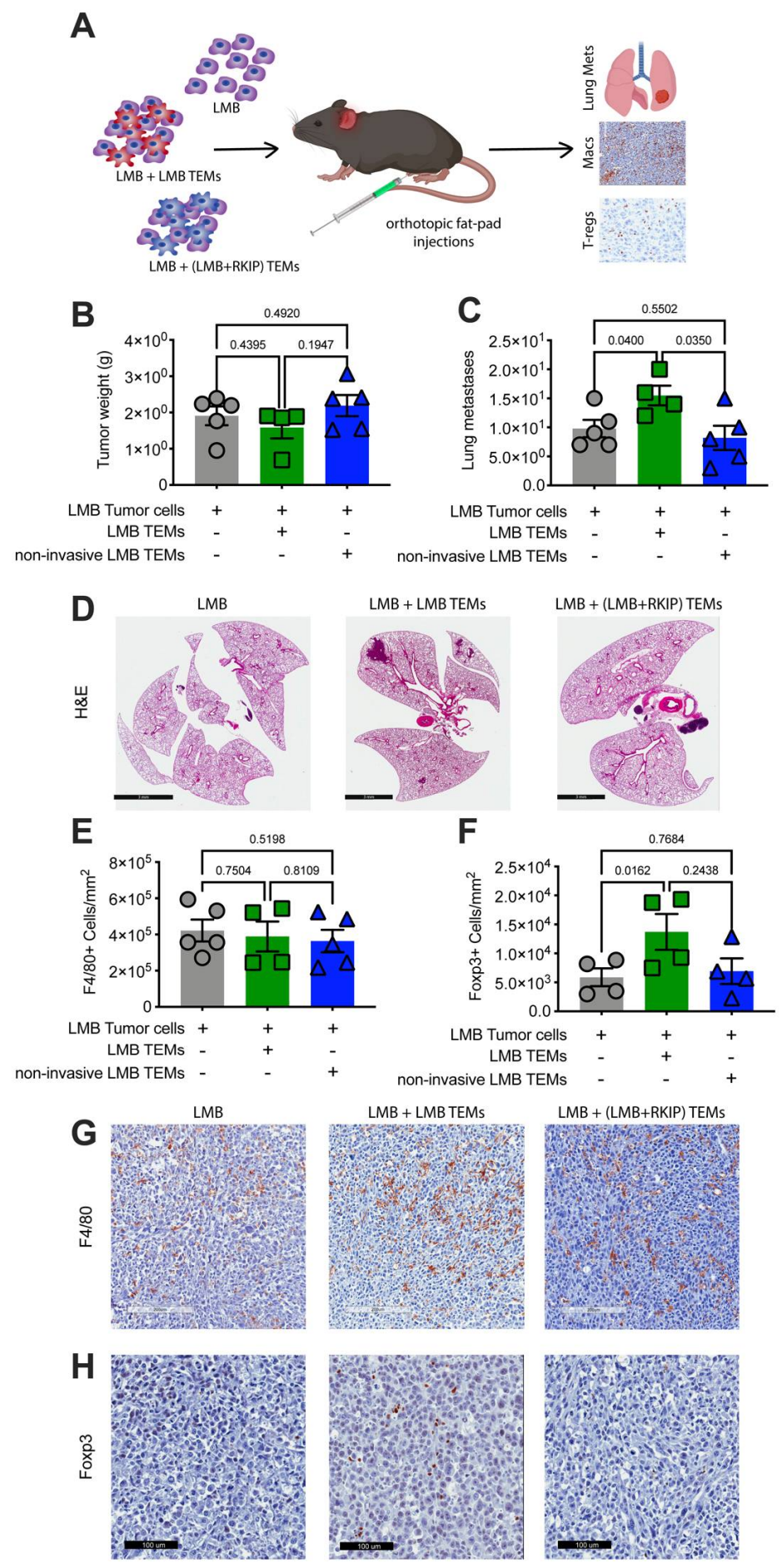

Figure 6. Tumor EVs promote metastasis through macrophages. (A) Either $0.5 \times 10^{6} \mathrm{LMB}$ EV programmed or LMB (overexpressing RKIP) EV programmed TEMs were co-injected with tumor cells as indicated. Final tumor weights (B) and number of metastases (C) per mouse are shown for LMB tumors alone (grey, $N=5$ ), LMB tumor cells with LMB programmed TEMs (green, $N=4$ ), and LMB tumor cells with LMB (RKIP overexpressing, non-metastatic) programmed TEMs (blue, $N=5$ ). (D) Representative images of lung metastases from each group. Final number of macrophages (E) and Foxp3+ T-regs (F) per mouse are shown for LMB tumors alone (grey, $N=5$ ), LMB tumor cells with LMB programmed TEMs (green, $N=4$ ), and LMB tumor cells with LMB (RKIP overexpressing, non-metastatic) programmed TEMs (blue, $N=5$ ). Representative images of $F 4 / 80+$ macrophages $(\mathbf{G})$ and Foxp3+ T-regs (H) from each group. (All $p$-values were calculated utilizing a $t$-test). 


\subsection{Clinical Significance of Tumor EV Macrophage Programming}

To evaluate the clinical significance of tumor EV programming of macrophages, we looked in our data for cytokines commonly regulated by tumor EV secretion and tumor CCL5 expression (Table S1; Figures 3B and 5C). DAVID analysis [49] of this gene set indicated enrichment in macrophage activation, chemotaxis, cell proliferation, negative regulation of apoptosis, and cell signaling (Figure S11). These genes in this set regulated both by EV numbers and tumor CCL5 expression are altered in a variety of solid tumor types, including melanoma, lung, bladder, ovarian, stomach, head and neck, uterine, breast, cervical, colorectal, liver, and prostate cancer as well as high- and low-grade gliomas (Figure 7A). Analysis of the expression of CCL5-regulated cytokines in patient breast tumors revealed $\sim 75$ percent were highly correlated with CCL5 expression in TCGA (Figure 7B). When we examined the expression of these CCL5-regulated cytokines across breast tumors, we found that high expression of cytokines and chemokines clustered with high expression of CCL5 in triple-negative/basal-like breast tumors (Figure 7C and Figure S12). Together, these results suggest that increased CCL5 expression in tumors leads to increased and altered EVs from tumor cells. These EVs then educate macrophages, which then secrete cytokines and other pro-metastatic factors that alter the tumor microenvironment and drive tumor metastasis (see summary schematic in Figure 7D).
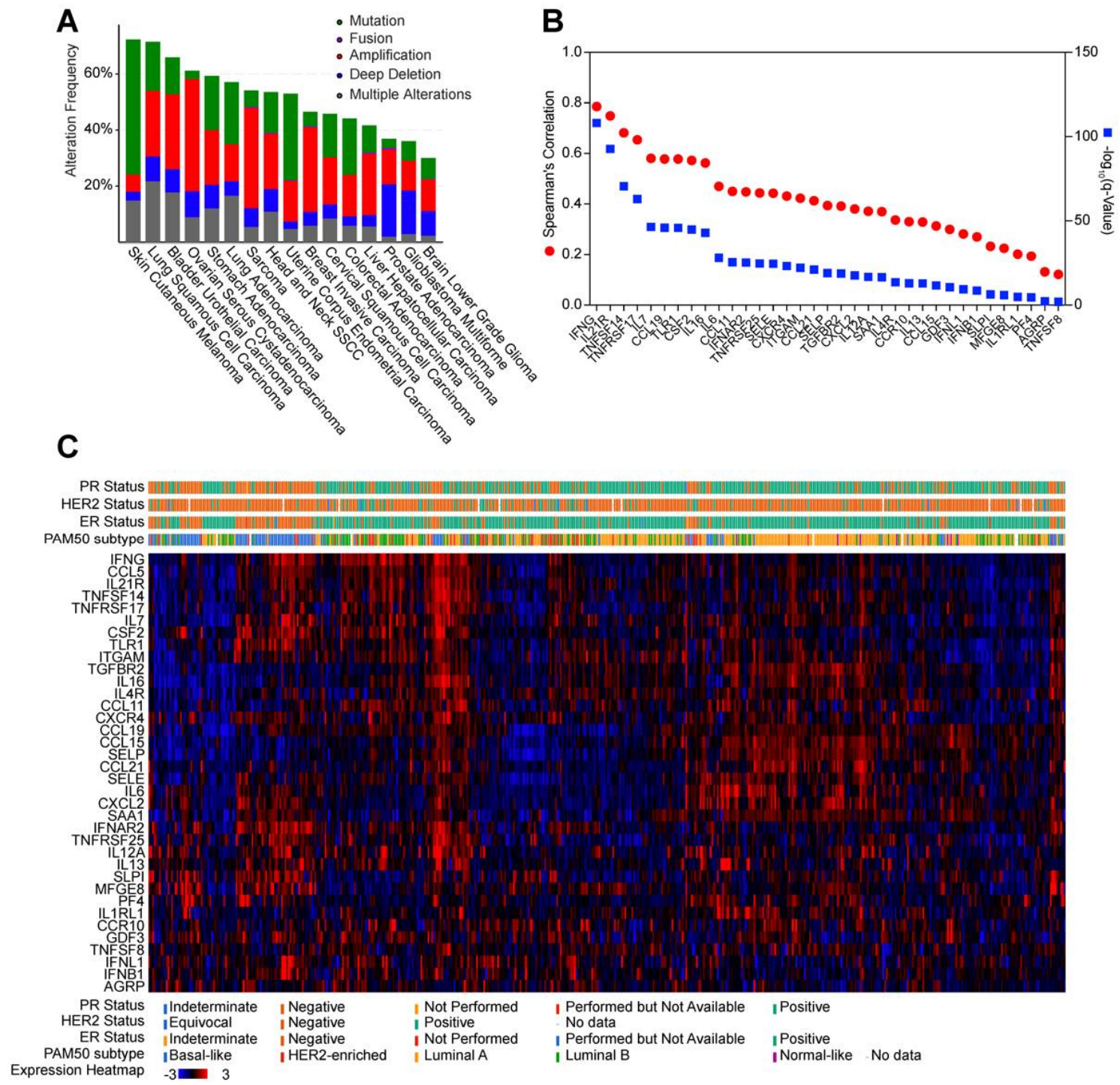

Figure 7. Cont. 


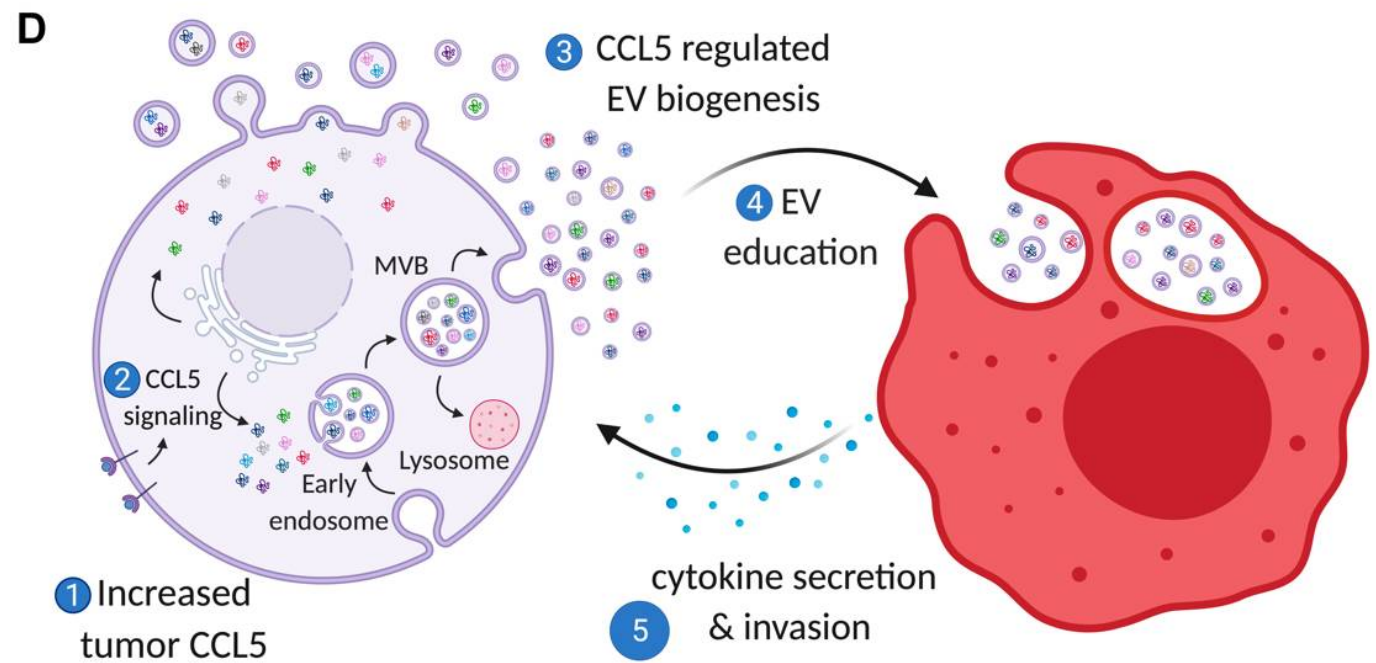

Figure 7. Clinical Significance of EVs as Biomarkers. (A) Top 16 tumor types with genetic alterations in genes we found differentially expressed in macrophages by tumor Rab27a and CCL5. (B) Spearman correlation of genes regulated in macrophages by tumor Rab27a and CCL5 to CCL5 in all TCGA breast samples $(N=1100)$ (C) Expression heatmap of TCGA breast cancer patients for mRNAs induced in macrophages by tumor Rab27a and CCL5. Patients were clustered by co-expression in cBioPortal, and ER/PR status by IHC is shown. (D) Schematic depicting tumor CCL5 expression regulating EV stimulation of macrophages leading to secretion of CCL7, CSF2, IL-21R, IL7, CXCR4, CCL19, IFNG, and OPN, increasing tumor metastasis.

\section{Discussion}

Here we demonstrate that tumor EVs are an integral component for education of macrophages capable of driving metastasis in TNBC. CCL5 regulates both the secretion of EVs from tumor cells, as well as their ability to program macrophages. Analyses of clinical breast cancer data showed significant correlation between expression of CCL5 and a set of genes correspondingly regulated in macrophages by tumor EVs and CCL5 expression. These genes were specifically enriched in TNBC or basal-like patients. The metastatic state of the tumor was translated to macrophages through EV programming, suggesting that the tumor EVs are capturing and transmitting the metastatic state of the tumor to the microenvironment.

This work also shows that CCL5 regulates not only macrophage recruitment but also programming. Studies by our group and others have demonstrated that CCL5 plays a critical role in the recruitment of TAMs that drive tumor metastasis $[10,11]$ as well as directly stimulating tumor progression [16]. By contrast, the present work shows that CCL5 protein secreted from tumors does not have a direct effect on macrophage programming to a TAM phenotype. Instead, these data suggest that CCL5 autocrine signaling promotes generation of tumor EVs that reprogram macrophages to a more metastatic, TAM function. Changing CCL5 expression in tumors alters cargo such as CCL5 mRNA in EVs as well as the number of EVs secreted. Additionally, our study shows that is not the number of macrophages recruited to the tumor, but their phenotype that is important for tumor metastasis. These findings further demonstrate that CCL5 plays a novel role, through EVs, in altering macrophage function rather than just recruitment. Macrophages altered by tumor CCL5 expression and EVs have increased expression of several genes known to drive invasion and metastasis, including OPN [44], SLPI [45-48], HGF [43], and NRG3 [50]. There are several possible reasons why activation by CCL5-stimulated EVs rather than direct CCL5 interaction is required to educate tumor-recruited macrophages. For example, even in the context of a single macrophage receptor, EVs can express multiple ligands and, therefore, may bind with higher affinity.

Several of the proteins that are regulated in TEMs and TAMs by tumor EVs and CCL5 are potential chemotactic factors for other cell types within the microenvironment such as Tregs. In particular, CCL19 is a chemotactic factor involved in recruiting CD4+CD25+CD69- 
T-regs to zones of $\mathrm{T}$ cell infiltration in the tumor micro-environment [51-53]. Expression of CXCL1 also recruits T-regs to the tumor microenvironment through CXCR2 signaling [54]. In addition, interferon- $\gamma$ (IFNG) has been implicated in T-reg polarization [55-58]. Since CCL19 and IFNG are highly correlated with CCL5 expression in patient breast tumors, these may be most relevant to T-reg infiltration of tumors in response to CCL5. Our data suggest that tumor EVs not only impact TAM phenotypes directly, but also alter their ability to attract and interact with other cells that contribute to evasion of immune detection.

These results highlight the importance of CCL5 and tumor EVs as key potential targets for tumor immunotherapy. Previous work has established that tumors infiltrated with $\mathrm{T}$ cells, T-regs, and macrophage recruitment may respond well to checkpoint blockade therapy $[59,60]$. The genes we see regulated in macrophages by CCL5 altered EVs are highly correlated with expression of CD8, CD4, CD25, LAG3, Foxp3, and IFNG, as well as macrophage markers, in TNBC patients. This suggests that tumor cells EV protein or RNA markers may also be prognostic for response to checkpoint blockade therapies. Because of the strong evidence of a biological role these EVs play in altering the immune microenvironment, it furthers their potential for use as biomarkers of tumor outcome as well as potential response to immunotherapy.

Overall, our results show that tumor-associated macrophages, which play a key role in enabling tumor cells to invade surrounding tissue, intravasate into vessels, and metastasize, rely on tumor EVs from metastatic cells to be programmed toward a prometastatic phenotype. As such, EVs can play an even more pivotal role in metastatic progression than previously realized. Targeting tumor EVs will not only be essential to reduce the formation of the pre-metastatic niche, but also block the education of the primary tumor microenvironment including macrophages to reduce tumor burden and tumor cell dissemination.

Supplementary Materials: The following are available online at https://www.mdpi.com/article/ 10.3390/cancers13143459/s1, Figure S1: Schematic describing our experimental design for in vitro EV analysis; Figure S2: Validation of small-EV markers by flow, immunoblot, and scanning EM; Figure S3: Reduction in EV secretion in BM1 and LMB cells upon Rab27a Knock-Down; Figure S4: Rab27a Knock-Down in BM1 cells does not alter tumor growth, migration, or invasion; Figure S5: Cytokine array analysis of EV-programmed macrophages produced from control and shRab27a tumor cells; Figure S6: CCL5 protein alone not sufficient to drive macrophage programming; Figure S7: CCL5 regulates EV secretion in BM1 and LMB tumor cells; Figure S8: Cytokine array analysis of macrophages programmed with either control or CCL5-Knock-Down EVs; Figure S9: CCL5 recruited TAMs increase both tumor cell growth and metastasis; Figure S10: Tumor RKIP expression does not alter EV secretion, but blocks pro-tumor programming of macrophages by tumor EVs; Figure S11: DAVID analysis macrophage genes regulated by EV programming and tumor CCL5 expression; Figure S12: Heatmap of z-score normalized gene expression in METABRICK individual patient breast cancer samples.

Author Contributions: Conceptualization, D.C.R. and M.R.R.; methodology, D.C.R. and M.R.R.; investigation \& data analysis, D.C.R., N.D.W., F.D.R., J.W. and J.L.; writing-original draft, D.C.R.; writing-review and editing, D.C.R., N.D.W., F.D.R., J.W., J.L., S.L.S. and M.R.R.; funding acquisition, D.C.R., S.L.S. and M.R.R.; resources, S.L.S. and M.R.R.; supervision, S.L.S. and M.R.R. All authors have read and agreed to the published version of the manuscript.

Funding: This study was funded by National Cancer Institute grants R01-CA184494 (to MRR), R01-CA226871 (to SLS), F31-CA192780 (to DCR), and in part by P30-CA014599 (to the University of Chicago Human Tissue Resource Center); and by the Rustandy fund for Innovative Cancer Research (to MRR). American Cancer Society (132030-RSG-18-108-01-TBG, SLS).

Institutional Review Board Statement: All mice were housed and handled according to the guidelines of and approved by the University of Chicago Institutional Animal Care and Use Committee guidelines (protocol 7228-8 June 2016).

Informed Consent Statement: Not applicable. 
Data Availability Statement: Data generated or analyzed during this study are included in this published article (and its supplementary information files). Reasonable requests for other raw data supporting this work can be made to Dr. Rosner.

Acknowledgments: The authors are particularly grateful to Melody Swartz for generously giving us access to her NanoSight NS300 microscope. The authors would also like to thank members of the Swartz and Gajewsky laboratories at the University of Chicago for helpful discussions. Biological illustrations created with BioRender.com (accessed on 2 June 2021).

Conflicts of Interest: The authors declare no conflict of interest. The funders had no role in the design of the study; in the collection, analyses, or interpretation of data; in the writing of the manuscript, or in the decision to publish the results.

\section{Appendix A}

BMDMs treated with $20 \mathrm{ng} / \mathrm{mL}$ of M-CSF served as a negative control (Figure S1). For these studies, we used the TNBC cell line BM1, a highly invasive bone-metastatic variant of MDA-MB-231 [36], or mouse TNBC cells, E0771-LMB (referred to as LMB) [37]. While there are many human lines for TNBC, we utilized BM1 cells because they are highly invasive and because their signaling pathway has been extensively studied by our lab. We previously showed that RKIP regulates CXCR4 and OPN through HMGA1 and BACH1 through the miRNA let-7 [61]. Additionally, in our previous work, we showed that RKIP regulated macrophage recruitment by downregulating CCL5 expression in BM1 cells [11]. LMB cells were utilized because of their highly invasive nature, as well as similarity to human TNBC expression compared to other syngeneic models [37]. EVs were enriched from the TNBC cell line BM1, a highly invasive bone-metastatic variant of MDA-MB-231 [36], or mouse TNBC cells, E0771-LMB (referred to as LMB) [37] using iZon qEV columns (Figure S2A-C). To determine how to best derive EVs, we tested whether EVs isolated from tumor cells ex vivo compared to in vitro or in serum free vs exosome depleted FBS (Gibco) altered macrophage biology. We found no difference between macrophages treated with EVs from ex vivo or in vitro tumor cells (Figure S2D) or between EVs isolated in serum free culture media compared to grown with media supplemented with exosome depleted FBS (Figure S2E). We moved forward with isolation of EVs in serum free media from in vitro tumor cells. BMDMs were treated with $20 \mathrm{ng} / \mathrm{mL} \mathrm{M-CSF}$ with $1 \times 10^{8} \mathrm{EVs} / \mathrm{mL}$ from BM1 tumor cells or $1 \times 10^{9} \mathrm{EVs} / \mathrm{mL}$ for LMB cells for $48 \mathrm{~h}$ to generate tumor EV-educated macrophages (TEMs). Once TEMs were educated, we used two assays to characterize TEMs. (1) RNA or secreted factors were isolated from TEMs and assayed for cytokine expression of pro-metastatic macrophage markers (Ccl7, Tnfr2, or Mmp12) [11,62,63]. (2) TEMs were washed and incubated in serum free media to assess the effect of TEM secreted factors in our invasion assay.

\section{Appendix B}

To block the metastatic ability of tumor cells, we overexpressed the metastasis suppressor gene Raf kinase inhibitor protein (RKIP, PEBP1). We have extensively demonstrated the ability of RKIP to block both early and late metastasis [38], regulate HMGA2 and $\mathrm{BACH1}$ signaling $[61,64]$, as well as regulate macrophage recruitment to TNBC through regulation of tumor CCL5 expression [11]. When we overexpressed RKIP in BM1 and LMB cells, we found no significant change in the number of EVs secreted from either cell line (Figure S10A-G). However, TEMs programmed from RKIP over-expressing cells were not able to significantly drive tumor cell invasion, while TEMs created with EVs from control cells were able to significantly drive invasion (Figure S10G-I). When we examined expression of pro-metastatic macrophage marker genes we previously established [11], we found that TEMs created with EVs from RKIP-overexpressing cells had significantly lower expression of Grn, Mmp12, and Ccl7 compared to TEMs created with EVs from control tumor cells (Figure S10I). Additionally, TEMs created with EVs from RKIP-overexpressing cells had similar levels of expression of Grn, Mmp12, and Ccl7 compared to TEMs created with EVs from the normal mammary epithelial cell line 184A1 (Figure S10I). 


\section{References}

1. Carey, L.A.; Perou, C.M.; Livasy, C.A.; Dressler, L.G.; Cowan, D.; Conway, K.; Karaca, G.; Troester, M.A.; Tse, C.K.; Edmiston, S.; et al. Race, breast cancer subtypes, and survival in the Carolina Breast Cancer Study. JAMA J. Am. Med. Assoc. 2006, 295, 2492-2502. [CrossRef]

2. Howlader, N.; Noone, A.; Krapcho, M.; Miller, D.; Bishop, K.; Altekruse, S.; Kosary, C.; Yu, M.; Ruhl, J.; Tatalovich, Z.; et al. SEER Cancer Statistics Review (CSR) 1975-2013; National Cancer Institute: Bethesda, MD, USA, 2016.

3. Anders, C.K.; Carey, L.A. Biology, metastatic patterns, and treatment of patients with triple-negative breast cancer. Clin. Breast Cancer 2009, 9 (Suppl. 2), S73-S81. [CrossRef]

4. Joyce, J.A.; Pollard, J.W. Microenvironmental regulation of metastasis. Nat. Rev. Cancer 2009, 9, 239-252. [CrossRef]

5. Green, C.E.; Liu, T.; Montel, V.; Hsiao, G.; Lester, R.D.; Subramaniam, S.; Gonias, S.L.; Klemke, R.L. Chemoattractant signaling between tumor cells and macrophages regulates cancer cell migration, metastasis and neovascularization. PLoS ONE 2009, 4, e6713. [CrossRef]

6. Lin, E.Y.; Nguyen, A.V.; Russell, R.G.; Pollard, J.W. Colony-stimulating factor 1 promotes progression of mammary tumors to malignancy. J. Exp. Med. 2001, 193, 727-740. [CrossRef] [PubMed]

7. Zhang, W.; Zhu, X.-D.; Sun, H.-C.; Xiong, Y.-Q.; Zhuang, P.-Y.; Xu, H.-X.; Kong, L.-Q.; Wang, L.; Wu, W.-Z.; Tang, Z.-Y. Depletion of tumor-associated macrophages enhances the effect of sorafenib in metastatic liver cancer models by antimetastatic and antiangiogenic effects. Clin. Cancer Res. 2010, 16, 3420-3430. [CrossRef] [PubMed]

8. Medrek, C.; Pontén, F.; Jirström, K.; Leandersson, K. The presence of tumor associated macrophages in tumor stroma as a prognostic marker for breast cancer patients. BMC Cancer 2012, 12, 306. [CrossRef] [PubMed]

9. Qian, B.-Z.; Li, J.; Zhang, H.; Kitamura, T.; Zhang, J.; Campion, L.R.; Kaiser, E.A.; Snyder, L.A.; Pollard, J.W. CCL2 recruits inflammatory monocytes to facilitate breast-tumour metastasis. Nature 2011, 475, 222-225. [CrossRef] [PubMed]

10. Robinson, S.C.; Scott, K.A.; Wilson, J.L.; Thompson, R.G.; Proudfoot, A.E.I.; Balkwill, F.R. A chemokine receptor antagonist inhibits experimental breast tumor growth. Cancer Res. 2003, 63, 8360-8365. [PubMed]

11. Frankenberger, C.; Rabe, D.; Bainer, R.; Sankarasharma, D.; Chada, K.; Krausz, T.; Gilad, Y.; Becker, L.; Rosner, M.R. Metastasis Suppressors Regulate the Tumor Microenvironment by Blocking Recruitment of Prometastatic Tumor-Associated Macrophages. Cancer Res. 2015, 75, 4063-4073. [CrossRef] [PubMed]

12. Lu, H.; Clauser, K.R.; Tam, W.L.; Fröse, J.; Ye, X.; Eaton, E.N.; Reinhardt, F.; Donnenberg, V.S.; Bhargava, R.; Carr, S.A.; et al. A breast cancer stem cell niche supported by juxtacrine signalling from monocytes and macrophages. Nat. Cell Biol. 2014, 16, 1-9. [CrossRef]

13. Su, S.; Liu, Q.; Chen, J.J.; Chen, J.J.; Chen, F.; He, C.; Huang, D.; Wu, W.; Lin, L.; Huang, W.; et al. A Positive Feedback Loop between Mesenchymal-like Cancer Cells and Macrophages Is Essential to Breast Cancer Metastasis. Cancer Cell 2014, 25, 605-620. [CrossRef] [PubMed]

14. Amon, S.; Meier-Abt, F.; Gillet, L.C.; Dimitrieva, S.; Theocharides, A.P.A.; Manz, M.G.; Aebersold, R. Sensitive Quantitative Proteomics of Human Hematopoietic Stem and Progenitor Cells by Data-independent Acquisition Mass Spectrometry. Mol. Cell Proteom. 2019, 18, 1454-1467. [CrossRef]

15. Chang, L.-Y.; Lin, Y.-C.; Mahalingam, J.; Huang, C.-T.; Chen, T.-W.T.-C.; Kang, C.-W.; Peng, H.-M.; Chu, Y.-Y.; Chiang, J.-M.; Dutta, A.; et al. Tumor-Derived Chemokine CCL5 Enhances TGF- $\beta$-Mediated Killing of CD8+ T Cells in Colon Cancer by T-Regulatory Cells. Cancer Res. 2012, 72, 1092-1102. [CrossRef]

16. Yamaguchi, M.; Takagi, K.; Narita, K.; Miki, Y.; Onodera, Y.; Miyashita, M.; Sasano, H.; Suzuki, T. Stromal CCL5 Promotes Breast Cancer Progression by Interacting with CCR3 in Tumor Cells. Int. J. Mol. Sci. 2021, 22, 1918. [CrossRef] [PubMed]

17. Kowal, J.; Tkach, M.; Théry, C. Biogenesis and secretion of exosomes. Curr. Opin. Cell Biol. 2014, 29, 116-125. [CrossRef] [PubMed]

18. Pan, B.T.; Teng, K.; Wu, C.; Adam, M.; Johnstone, R.M. Electron microscopic evidence for externalization of the transferrin receptor in vesicular form in sheep reticulocytes. J. Cell Biol. 1985, 101, 942-948. [CrossRef]

19. Harding, C.; Heuser, J.; Stahl, P. Receptor-mediated endocytosis of transferrin and recycling of the transferrin receptor in rat reticulocytes. J. Cell Biol. 1983, 97, 329-339. [CrossRef]

20. Kalluri, R. The Biology and Function of Exosomes in Cancer. J. Clin. Invest. 2016, 126, 1208-1215. [CrossRef]

21. Théry, C.; Boussac, M.; Véron, P.; Ricciardi-Castagnoli, P.; Raposo, G.; Garin, J.; Amigorena, S. Proteomic analysis of dendritic cell-derived exosomes: A secreted subcellular compartment distinct from apoptotic vesicles. J. Immunol. 2001, 166, 7309-7318. [CrossRef]

22. Ostrowski, M.; Carmo, N.B.; Krumeich, S.; Fanget, I.; Raposo, G.; Savina, A.; Moita, C.F.; Schauer, K.; Hume, A.N.; Freitas, R.P.; et al. Rab27a and Rab27b control different steps of the exosome secretion pathway. Nat. Cell Biol. 2010, 12, 19-30. [CrossRef]

23. Park, M.; Jürgens, G. Membrane traffic and fusion at post-Golgi compartments. Front. Plant Sci. 2011, 2, 111. [CrossRef]

24. Webber, J.; Yeung, V.; Clayton, A. Extracellular vesicles as modulators of the cancer microenvironment. Semin. Cell Dev. Biol. 2015, 40, 27-34. [CrossRef]

25. Peinado, H.; Zhang, H.; Matei, I.R.; Costa-Silva, B.; Hoshino, A.; Rodrigues, G.; Psaila, B.; Kaplan, R.N.; Bromberg, J.F.; Kang, Y.; et al. Pre-metastatic niches: Organ-specific homes for metastases. Nat. Rev. Cancer 2017, 17, 302-317. [CrossRef] [PubMed]

26. Peinado, H.; Alečković, M.; Lavotshkin, S.; Matei, I.; Costa-Silva, B.; Moreno-Bueno, G.; Hergueta-Redondo, M.; Williams, C.; García-Santos, G.; Ghajar, C.M.; et al. Melanoma exosomes educate bone marrow progenitor cells toward a pro-metastatic phenotype through MET. Nat. Med. 2012, 18, 883-891. [CrossRef] [PubMed] 
27. Webber, J.; Steadman, R.; Mason, M.D.; Tabi, Z.; Clayton, A. Cancer Exosomes Trigger Fibroblast to Myofibroblast Differentiation. Cancer Res. 2010, 70, 9621-9630. [CrossRef] [PubMed]

28. Clayton, A. Cancer cells use exosomes as tools to manipulate immunity and the microenvironment. Oncoimmunology 2012, 1, 78-80. [CrossRef] [PubMed]

29. Liu, C.; Yu, S.; Zinn, K.; Wang, J.; Zhang, L.; Jia, Y.; Kappes, J.C.; Barnes, S.; Kimberly, R.P.; Grizzle, W.E.; et al. Murine mammary carcinoma exosomes promote tumor growth by suppression of NK cell function. J. Immunol. 2006, 176, 1375-1385. [CrossRef] [PubMed]

30. Szajnik, M.; Czystowska, M.; Szczepanski, M.J.; Mandapathil, M.; Whiteside, T.L. Tumor-Derived Microvesicles Induce, Expand and Up-Regulate Biological Activities of Human Regulatory T Cells (Treg). PLoS ONE 2010, 5, e11469. [CrossRef]

31. Luga, V.; Zhang, L.; Viloria-Petit, A.M.; Ogunjimi, A.A.; Inanlou, M.R.; Chiu, E.; Buchanan, M.; Hosein, A.N.; Basik, M.; Wrana, J.L. Exosomes mediate stromal mobilization of autocrine Wnt-PCP signaling in breast cancer cell migration. Cell 2012, 151, 1542-1556. [CrossRef]

32. Boelens, M.C.; Wu, T.J.; Nabet, B.Y.; Xu, B.; Qiu, Y.; Yoon, T.; Azzam, D.J.; Twyman-Saint Victor, C.; Wiemann, B.Z.; Ishwaran, H.; et al. Exosome Transfer from Stromal to Breast Cancer Cells Regulates Therapy Resistance Pathways. Cell 2014, 159, 499-513. [CrossRef]

33. Kapsogeorgou, E.K.; Abu-Helu, R.F.; Moutsopoulos, H.M.; Manoussakis, M.N.; Paredes, P.T.; Bossios, A.; Sjöstrand, M.; Gabrielsson, S.; Lötvall, J.; Valadi, H. Salivary gland epithelial cell exosomes: A source of autoantigenic ribonucleoproteins. Arthritis Rheum. 2005, 52, 1517-1521. [CrossRef]

34. Feng, D.; Zhao, W.-L.; Ye, Y.-Y.; Bai, X.-C.; Liu, R.-Q.; Chang, L.-F.; Zhou, Q.; Sui, S.-F. Cellular Internalization of Exosomes Occurs Through Phagocytosis. Traffic 2010, 11, 675-687. [CrossRef]

35. Costa-Silva, B.; Aiello, N.M.; Ocean, A.J.; Singh, S.; Zhang, H.; Thakur, B.K.; Becker, A.; Hoshino, A.; Mark, M.T.; Molina, H.; et al Pancreatic cancer exosomes initiate pre-metastatic niche formation in the liver. Nat. Cell Biol. 2015, 17, 816-826. [CrossRef]

36. Kang, Y.; Siegel, P.; Shu, W.; Drobnjak, M.; Kakonen, S.; Carlos, C.-C.; Guise, T.; Massagué, J. A multigenic program mediating breast cancer metastasis to bone. Cancer Cell 2003, 3, 537-549. [CrossRef]

37. Johnstone, C.N.; Smith, Y.E.; Cao, Y.; Burrows, A.D.; Cross, R.S.N.; Ling, X.; Redvers, R.P.; Doherty, J.P.; Eckhardt, B.L.; Natoli, A.L.; et al. Functional and molecular characterisation of EO771.LMB tumours, a new C57BL/6-mouse-derived model of spontaneously metastatic mammary cancer. Dis. Model. Mech. 2015, 8, 237-251. [CrossRef]

38. Surabhi, D.-G.; Yun, J.; Eves, E.M.; Newman, M.; Erkeland, S.J.; Hammond, S.M.; Minn, A.J.; Rosner, M.R.; Dangi-Garimella, S.; Yun, J.; et al. Raf kinase inhibitory protein suppresses a metastasis signalling cascade involving $\{$ LIN28\} and let-7. EMBO J. 2009, 28, 347-358. [CrossRef]

39. Théry, C.; Witwer, K.W.; Aikawa, E.; Alcaraz, M.J.; Anderson, J.D.; Andriantsitohaina, R.; Antoniou, A.; Arab, T.; Archer, F.; Atkin-Smith, G.K.; et al. Minimal information for studies of extracellular vesicles 2018 (MISEV2018): A position statement of the International Society for Extracellular Vesicles and update of the MISEV2014 guidelines. J. Extracell. Vesicles 2018, 7, 1535750. [CrossRef]

40. Bobrie, A.; Krumeich, S.; Reyal, F.; Recchi, C.; Moita, L.F.; Seabra, M.C.; Ostrowski, M.; Thery, C. Rab27a Supports ExosomeDependent and -Independent Mechanisms That Modify the Tumor Microenvironment and Can Promote Tumor Progression. Cancer Res. 2012, 72, 4920-4930. [CrossRef]

41. Shultz, L.D.; Ishikawa, F.; Greiner, D.L. Humanized mice in translational biomedical research. Nat. Rev. Immunol. 2007, 7, 118-130. [CrossRef]

42. Clarke, R. Human breast cancer cell line xenografts as models of breast cancer-The immunobiologies of recipient mice and the characteristics of several tumorigenic cell lines. Breast Cancer Res. Treat. 1996, 39, 69-86. [CrossRef] [PubMed]

43. Cecchi, F.; Rabe, D.; Bottaro, D. Targeting the $\{$ HGF/Met $\}$ signalling pathway in cancer. Eur. J. Cancer 2010, 46, 1260-1270. [CrossRef] [PubMed]

44. McAllister, S.S.; Gifford, A.M.; Greiner, A.L.; Kelleher, S.P.; Saelzler, M.P.; Ince, T.A.; Reinhardt, F.; Harris, L.N.; Hylander, B.L.; Repasky, E.A.; et al. Systemic Endocrine Instigation of Indolent Tumor Growth Requires Osteopontin. Cell 2008, 133, $994-1005$. [CrossRef]

45. Rasool, N.; LaRochelle, W.; Zhong, H.; Ara, G.; Cohen, J.; Kohn, E.C. Secretory leukocyte protease inhibitor antagonizes paclitaxel in ovarian cancer cells. Clin. Cancer Res. 2010, 16, 600-609. [CrossRef]

46. Sayers, K.T.; Brooks, A.D.; Sayers, T.J.; Chertov, O. Increased Secretory Leukocyte Protease Inhibitor (SLPI) Production by Highly Metastatic Mouse Breast Cancer Cells. PLoS ONE 2014, 9, e104223. [CrossRef]

47. Zhu, J.; Nathan, C.; Jin, W.; Sim, D.; Ashcroft, G.S.; Wahl, S.M.; Lacomis, L.; Erdjument-bromage, H.; Tempst, P.; Wright, C.D.; et al. Conversion of Proepithelin to Epithelins: Roles of SLPI and Elastase in Host Defense and Wound Repair. Cell 2002, 111, 867-878. [CrossRef]

48. Cui, C.; Chakraborty, K.; Tang, X.A.; Zhou, G.; Schoenfelt, K.Q.; Becker, K.M.; Hoffman, A.; Chang, Y.-F.; Blank, A.; Reardon, C.A.; et al. Neutrophil elastase selectively kills cancer cells and attenuates tumorigenesis. Cell 2021, 184, 3163-3177.e21. [CrossRef]

49. Huang, D.W.; Sherman, B.T.; Lempicki, R.A. Systematic and integrative analysis of large gene lists using DAVID bioinformatics resources. Nat. Protoc. 2009, 4, 44-57. [CrossRef]

50. Arteaga, C.L.; Engelman, J.A. ERBB receptors: From oncogene discovery to basic science to mechanism-based cancer therapeutics. Cancer Cell 2014, 25, 282-303. [CrossRef] 
51. Lim, H.W.; Hillsamer, P.; Kim, C.H. Regulatory T cells can migrate to follicles upon T cell activation and suppress GC-Th cells and GC-Th cell-driven B cell responses. J. Clin. Investig. 2004, 114, 1640-1649. [CrossRef]

52. Wei, S.; Kryczek, I.; Zou, W. Regulatory T-cell compartmentalization and trafficking. Blood 2006, 108, 426-431. [CrossRef]

53. Guerin, L.R.; Moldenhauer, L.M.; Prins, J.R.; Bromfield, J.J.; Hayball, J.D.; Robertson, S.A. Seminal Fluid Regulates Accumulation of FOXP3+ Regulatory T Cells in the Preimplantation Mouse Uterus Through Expanding the FOXP3+ Cell Pool and CCL19Mediated Recruitment1. Biol. Reprod. 2011, 85, 397-408. [CrossRef]

54. Lv, M.; Xu, Y.; Tang, R.; Ren, J.; Shen, S.; Chen, Y.; Liu, B.; Hou, Y.; Wang, T. miR141-CXCL1-CXCR2 Signaling-Induced Treg Recruitment Regulates Metastases and Survival of Non-Small Cell Lung Cancer. Mol. Cancer Ther. 2014, 13, 3152-3162. [CrossRef]

55. Wang, Z.; Hong, J.; Sun, W.; Xu, G.; Li, N.; Chen, X.; Liu, A.; Xu, L.; Sun, B.; Zhang, J.Z. Role of IFN-gamma in induction of Foxp3 and conversion of CD4+ CD25- T cells to CD4+ Tregs. J. Clin. Investig. 2006, 116, 2434-2441. [CrossRef] [PubMed]

56. Wood, K.J.; Sawitzki, B. Interferon $\gamma$ : A crucial role in the function of induced regulatory T cells in vivo. Trends Immunol. 2006, 27, 183-187. [CrossRef] [PubMed]

57. Zaidi, M.R.; Merlino, G. The Two Faces of Interferon- in Cancer. Clin. Cancer Res. 2011, 17, 6118-6124. [CrossRef] [PubMed]

58. Mandai, M.; Hamanishi, J.; Abiko, K.; Matsumura, N.; Baba, T.; Konishi, I. Dual Faces of IFNg in Cancer Progression: A Role of PD-L1 Induction in the Determination of Pro- and Antitumor Immunity CME Staff Planners' Disclosures Learning Objectives. Clin. Cancer Res. 2016, 22, 2329-2334. [CrossRef]

59. Woo, S.-R.; Corrales, L.; Gajewski, T.F. The STING pathway and the T cell-inflamed tumor microenvironment. Trends Immunol. 2015, 36, 250-256. [CrossRef]

60. Riaz, N.; Havel, J.J.; Makarov, V.; Desrichard, A.; Urba, W.J.; Sims, J.S.; Hodi, F.S.; Martín-Algarra, S.; Mandal, R.; Sharfman, W.H.; et al. Tumor and Microenvironment Evolution during Immunotherapy with Nivolumab. Cell 2017, 171, 934-949.e15. [CrossRef]

61. Yun, J.; Frankenberger, C.A.; Kuo, W.-L.; Boelens, M.C.; Eves, E.M.; Cheng, N.; Liang, H.; Li, W.; Ishwaran, H.; Minn, A.J.; et al. Signalling pathway for $\{$ RKIP $\}$ and Let-7 regulates and predicts metastatic breast cancer. EMBO J. 2011, 30, 4500-4514. [CrossRef]

62. Mishra, P.; Banerjee, D.; Adit, B.-B. Chemokines at the crossroads of tumor-fibroblast interactions that promote malignancy. J. Leukoc. Biol. 2011, 89, 31-39. [CrossRef] [PubMed]

63. Jung, D.-W.; Che, Z.M.; Kim, J.; Kim, K.; Kim, K.-Y.; Williams, D.; Kim, J. Tumor-stromal crosstalk in invasion of oral squamous cell carcinoma: A pivotal role of CCL7. Int. J. Cancer 2010, 127, 332-344. [CrossRef] [PubMed]

64. Lee, J.; Lee, J.; Farquhar, K.S.; Yun, J.; Frankenberger, C.A.; Bevilacqua, E.; Yeung, K.; Kim, E.-J.; Balázsi, G.; Rosner, M.R. Network of mutually repressive metastasis regulators can promote cell heterogeneity and metastatic transitions. Proc. Natl. Acad. Sci. USA 2014, 111, E364-E373. [CrossRef] [PubMed] 